\title{
Characterisation of denatured states of sensory rhodopsin II by solution-state NMR
}

Yi Lei $\operatorname{Tan}^{1}$, James Mitchell ${ }^{2,3}$, Judith Klein-Seetharaman ${ }^{2,4}$, Daniel Nietlispach ${ }^{{ }^{*}}$

${ }^{1}$ Department of Biochemistry, 80 Tennis Court Road, University of Cambridge, CB2 1GA, United Kingdom

${ }^{2}$ Biomedical Sciences Division, Warwick Medical School, University of Warwick, Coventry, CV4 7AL, United Kingdom

${ }^{3}$ Present address: School of Life Sciences, University of Nottingham, NG7 2UH, United Kingdom

${ }^{4}$ Present address: Department of Chemistry, Integrative Graduate Program in Quantitative Biosciences and Engineering, Colorado School of Mines, 1500 Illinois Street, Golden CO 80401

*To whom correspondence should be addressed: Daniel Nietlispach, Department of Biochemistry, 80 Tennis Court Road, University of Cambridge, CB2 1GA, United Kingdom. Phone: +44 1223 766023, Fax: +44 1223 766002, Email: dn206@cam.ac.uk 


\begin{abstract}
Sensory rhodopsin II (pSRII), a retinal-binding photophobic receptor from Natronomonas pharaonis, is a novel model system for membrane protein folding studies. Recently, the SDSdenatured states and the kinetics for reversible unfolding of pSRII have been investigated, opening the door to the first detailed characterisation of denatured states of a membrane protein by solution-state nuclear magnetic resonance (NMR) using uniformly ${ }^{15} \mathrm{~N}$-labelled pSRII. SDS denaturation and acid denaturation of pSRII both lead to fraying of helix ends but otherwise small structural changes in the transmembrane domain, consistent with little changes in secondary structure and disruption of the retinal-binding pocket and tertiary structure. Widespread changes in the backbone amide dynamics are detected in the form of line broadening, indicative of $\mu$ s-to-ms timescale conformational exchange in the transmembrane region. Detailed analysis of chemical shift and intensity changes lead to high-resolution molecular insights on structural and dynamics changes in SDS- and aciddenatured pSRII, thus highlighting differences in the unfolding pathways under the two different denaturing conditions. These results will form the foundation for furthering our understanding on the folding and unfolding pathways of retinal-binding proteins and membrane proteins in general, and also for investigating the importance of ligand-binding in the folding pathways of other ligand-binding membrane proteins, such as GPCRs.
\end{abstract}

\title{
Highlights
}

- We report detailed NMR studies on a denatured polytopic $\alpha$-helical membrane protein

- SDS- and acid-denatured pSRII experience small structural changes in the TM domains

- Denatured pSRII undergoes widespread $\mu s$-to-ms timescale conformational exchange

- High-resolution structural differences between SDS vs. acid denaturation are shown

- Retinal influences the exchange equilibria amongst different conformations of pSRII

\section{Keywords}

Membrane proteins; Folding; Transmembrane helices; Chemical shifts; Backbone amides

\author{
Abbreviations \\ 7TM Seven transmembrane \\ $X_{\text {SDS }} \quad$ Mole fraction of SDS \\ BEST Band-selective excitation short transient \\ c7-DHPC Diheptanoylphosphatidylcholine; 1,2-diheptanoyl-sn-glycero-3-phosphocholine \\ CD Circular dichroism \\ $\mathrm{H}$-bond Hydrogen bond \\ HSQC Heteronuclear single quantum correlation \\ MRE Mean residue ellipticity \\ NMR Nuclear magnetic resonance \\ pKa N Negative base-10 logarithm of the acid dissociation constant \\ pSRII Natronomonas pharaonis sensory rhodopsin II
}


SDS

Sodium dodecyl sulphate

TM Transmembrane

TROSY Transverse relaxation optimised spectroscopy

TSP Trimethylsilylpropanoic acid

UV/vis UV/visible 


\section{Introduction}

Our understanding on the folding of membrane proteins lags behind that of soluble proteins due to challenges posed by the exposure of hydrophobic regions leading to aggregation during in vitro chemical denaturation and refolding experiments. While different folding models are accepted for soluble proteins, only the two-stage model based on studies with bacteriorhodopsin [1-9] and the long-range interactions model based on studies with mammalian rhodopsin [10-14] have been proposed so far for helical membrane proteins. Recently, sensory rhodopsin II (pSRII) has emerged as a new model system for membrane protein folding studies [15]. It is a retinal-binding seven transmembrane (7TM) helical membrane protein from the archaeon Natronomonas pharaonis, and functions as a repellent phototactic receptor to blue light via photoisomerisation of its all-trans retinal chromophore to the 13-cis conformation. This enables the archaeon to seek the dark when respiratory substrates are plentiful. Simulated thermal unfolding experiments suggest that pSRII is stabilised by a combination of mechanisms found for rhodopsin and bacteriorhodopsin [16], with a subset of helices breaking off early in the simulation and a core involving residues from other helices and loops remaining until late, suggesting the presence of a folding core. A systematic investigation of the structural features of SDS-denatured states and the kinetics for reversible unfolding of pSRII has shown that the protein is difficult to denature, and retains the equivalent of six out of seven transmembrane (TM) helices even in $30 \%$ SDS $\left(0.998 X_{\text {SDS }}\right.$, where $X_{\text {SDS }}$ denotes the mole fraction of SDS) while the retinal-binding pocket is disrupted in low SDS concentrations starting from $1.5 \%$ SDS $\left(0.834 X_{\text {SDS }}\right)$ [15]. By extensively characterising SDS-denatured states and the kinetics of unfolding and refolding pSRII from SDS-denatured states, we have demonstrated pSRII as a new model membrane protein which is suitable for protein folding studies. Refolding of pSRII has a unique folding mechanism that differs from those of bacteriorhodopsin and bovine rhodopsin. While refolding of SDS-denatured bacteriorhodopsin with bound retinal adheres to two-state folding kinetics $[17,18]$, the refolding kinetics of the equivalent retinal-bound state of SDS-denatured pSRII showed clear deviations from the two-state folding regime [15]. pSRII and bacteriorhodopsin can both also be refolded from the SDS-denatured apo protein state devoid of retinal chromophore $[15,17]$. On the other hand, refolding of bovine rhodopsin remains elusive even from partially denatured states [19].

More experimental studies are needed to understand the folding pathway of membrane proteins, particularly how and when secondary and tertiary structure elements are being formed. Retinal-binding 7TM helical proteins such as bacteriorhodopsin, mammalian rhodopsin and now pSRII are excellent models for membrane protein folding studies, since the retinal chromophore acts as a natural reporter of the retinal-binding pocket and thus, the tertiary structure. Herein, we report in-depth characterisation of pSRII under denaturing conditions by NMR spectroscopy. Solution-state NMR offers distinct advantages in providing structure and dynamics information at atomic resolution for highly-populated ground states as well as transient minor populations in bio-macromolecules [20,21]. A range of NMR methods are available for studying protein folding events which occur at different timescales and for probing the properties of low-population folding intermediates, hence yielding detailed insights into folding landscapes, misfolding, aggregation and function [2228]. Comprehensive studies on the denatured states of full-length polytopic $\alpha$-helical 
membrane proteins are scarce [29], and have mostly been limited to sparsely-labelled samples [30-33] or fragments of polytopic $\alpha$-helical membrane proteins [34-36]. Insights on unfolding pathways and unfolded states could only be derived from a small subset of amino acids or chemical groups in the protein. Multiple challenges are involved in detailed NMR studies on protein folding, including (1) the requirement of uniformly-labelled denatured membrane protein samples which remain resistant against sample degradation and aggregation over several days to enable multi-dimensional NMR studies, (2) successfully assigning a high proportion of NMR resonances due to potential challenges posed by spectral overlap, and (3) studying a heterogeneous ensemble of unfolded structures with different backbone dynamics and conformational exchange occurring at different timescales.

In this paper, we describe the characterisation of SDS-denatured pSRII and acid-denatured pSRII by means of $1 D$ and 2D solution-state NMR. Small changes in the chemical environment of backbone amides were detected, consistent with previous results showing that denatured pSRII retains most of its helical content and remains embedded in detergent micelles [15]. Changes in backbone amide dynamics, specifically increased observation of conformational exchange on the $\mu$ s-to-ms timescale, was an important feature of SDS- and acid-denatured pSRII. This paper presents one of the very few [29] detailed backbone NMR studies on a full-length, denatured polytopic $\alpha$-helical membrane protein, thereby providing high-resolution molecular insights on differences in the unfolding pathways under different denaturing conditions. Since the absence of native retinal-protein interactions in denatured states enables the backbone amides to sample different conformations, it is further proposed that the formation of native retinal-protein interactions during protein folding might influence the equilibrium amongst different conformations sampled by the backbone amides, and, in turn, the protein folding landscape.

\section{Results}

\section{Structural changes during SDS denaturation of pSRII initiated from helix C}

Backbone amide chemical shifts are sensitive to hydrogen bonds ( $\mathrm{H}$-bonds) and secondary structures of proteins $[37,38]$. Chemical shift changes can therefore be used to deduce structural information and changes in $\mathrm{H}$-bonding. The denaturation of pSRII in increasing concentrations of SDS was investigated by recording 2D $\left[{ }^{1} \mathrm{H},{ }^{15} \mathrm{~N}\right]$ BEST-TROSY spectra and monitoring changes in the backbone amide chemical shifts.

To understand whether backbone amide chemical shifts are determined by the mole fraction of SDS $\left(X_{S D S}\right)$ or the absolute concentration of SDS, 2D $\left[{ }^{1} \mathrm{H},{ }^{15} \mathrm{~N}\right]$ BEST-TROSY spectra of pSRII were recorded at $308 \mathrm{~K}$ in $1 \%$ SDS + 0.45\% c7-DHPC and in $2 \%$ SDS + 0.90\% c7-DHPC, both of which correspond to $0.769 X_{\text {SDS }}$ but with different molar concentrations of c7-DHPC and SDS (Figure S1). The retinal chromophore remained attached under both conditions, indicating that both conditions were below the unfolding transition, which previously was determined to be $\sim 0.81 X_{\text {SDS }}$ in the presence of 0.5\% c7-DHPC [15]. Virtually identical chemical shifts were observed in both spectra, indicating that chemical shifts are determined by $X_{\text {SDS }}$. The peak intensities were slightly lower in $2 \%$ SDS $+0.90 \%$ c7-DHPC due 
to higher detergent concentrations, leading to higher viscosity, slower molecular tumbling and broader linewidths.

Folded pSRII $\left(0 X_{\text {SDS }}\right.$ ) showed wide chemical shift dispersion $[39,40]$. The dispersion remained equally wide in the presence of increasing $X_{\mathrm{SDS}}$ (Figure 1a), indicating that many backbone amides still experience unique chemical environments under denaturing conditions. This supports the results from our previous circular dichroism experiments, which show that large portions of helical structure are preserved across all $X_{\text {SDS }}$ studied [15].

The trajectories of peak movements in titrations monitored by chemical shift changes provide insights on protein-ligand interactions. Linear changes in resonance positions in the titration plot indicate the presence of only two states, the free and the bound states, in fast exchange on the chemical shift timescale with each other. Secondary interactions (e.g. alternative ligand binding site) and conformational changes during the titration lead to nonlinear changes in resonance positions, because the protein would be expected to interact differently with the ligand compared to the primary interaction $[41,42]$. An overlay of $\left[{ }^{1} \mathrm{H},{ }^{15} \mathrm{~N}\right]$ BEST-TROSY spectra recorded in 0-0.990 $X_{\text {SDS }}(0-30 \%$ SDS in 0.5\% c7-DHPC) shows that increasing $X_{S D S}$ led to progressive changes in chemical shifts (see arrows in Figure $1 \mathrm{~b}$ ). Many backbone amides showed non-linear changes in resonance position with increasing $X_{\text {SDS }}$ (Figure 1c), and these residues were found to spread across all seven TM helices (Figure $1 d)$. This suggests that in addition to the SDS-free and SDS-bound states, many backbone amide environments sample alternative states which are attributable to small but widespread conformational changes across the TM helical bundle as backbone amides interact with SDS and as the protein denatures in the presence of high $X_{\text {SDS. The extent at }}$ which peak movements deviate from linearity depends on the relative populations of the different fast-exchanging states present. This indicates that the number of residues with alternative states presented in Figure $1 \mathrm{~d}$ is likely to be an underestimation, as very minor populations of alternative states would not be readily detectable with this method.

To obtain a systematic understanding on the structural changes of pSRII upon exposure to SDS, the combined chemical shift difference $\left(\Delta \delta\left({ }^{1} \mathrm{H},{ }^{15} \mathrm{~N}\right)\right.$; see Materials and Methods) relative to $\mathrm{pSRII}$ in $0 X_{S \mathrm{SS}}$ was calculated for each observable backbone amide resonance across different $X_{\text {SDS }}$ (Figure 2a). In general, $\Delta \delta\left({ }^{1} H,{ }^{15} \mathrm{~N}\right.$ ) increased with increasing $X_{\mathrm{SDS}}$, with residues near the ends of helices showing greater $\Delta \delta\left({ }^{1} \mathrm{H},{ }^{15} \mathrm{~N}\right)$. This suggests that the ends of helices were unwinding slightly (fraying) whereas the helical content in the protein core was preserved, consistent with the observed high preservation of helical content across the wide range of $X_{S D S}$ previously reported in our CD studies [15].

To determine how the retinal-binding pocket is disrupted during SDS denaturation, residues which constitute the retinal-binding pocket are identified by taking a $3 \AA$ cut-off around the retinal chromophore (Figure $2 \mathrm{~b}$ ). These residues are listed in Table 1 , and are consistent with the retinal-binding pocket residues identified in the X-ray crystallography study by Royant et al $[43,44]$. In $0-0.625 X_{\text {SDS }}(0-0.5 \%$ SDS in $0.5 \%$ C7-DHPC), residues in the retinalbinding pocket had $\Delta \delta\left({ }^{1} \mathrm{H}^{15} \mathrm{~N}\right)<0.1 \mathrm{ppm}$ and experienced little perturbation of the surrounding chemical environment, with the exception of Trp-178, which is located near the extracellular end of helix $\mathrm{F}$ and reports on slight unravelling of helix ends. Starting from Trp76 in the middle of helix $C$ at $\geq 0.625 X_{\text {SDS }}(0.5 \%$ SDS in $0.5 \%$ c7-DHPC), residues with 
$\Delta \delta\left({ }^{1} \mathrm{H},{ }^{15} \mathrm{~N}\right)>0.1 \mathrm{ppm}$ gradually spread across helix C (Trp-76, Thr-79, lle-83) and helix D (Val108 , Met-109) with increasing $X_{\text {SDS. }}$. These observations suggest loosened packing of helices $C$ and $D$ against the retinal-binding pocket even before the unfolding transition, which was previously determined to be $\sim 0.81 X_{\text {SDS }}$ in the presence of $0.5 \% \mathrm{c7}$-DHPC [15]. From 0.728 $X_{\text {SDS }}\left(0.8 \%\right.$ SDS in $0.5 \% \mathrm{c} 7$-DHPC) onwards, $\Delta \delta\left({ }^{1} \mathrm{H},{ }^{15} \mathrm{~N}\right)>0.1 \mathrm{ppm}$ also appeared on helix $\mathrm{G}$ (Phe-208, Gly-209) at one helical turn away from the site of retinal attachment (Lys-205), whereas Lys-205 showed $\Delta \delta\left({ }^{1} \mathrm{H},{ }^{15} \mathrm{~N}\right)<0.1 \mathrm{ppm}$ across all $X_{\mathrm{SDS}}$. This suggests that the site of Schiff base attachment and nearby residues experience small changes to backbone $\mathrm{H}$ bonding and secondary structure beyond the unfolding transition ( $>0.81 X_{\text {SDS }}$ ). In summary, SDS denaturation leads to disruption of the retinal-binding pocket, initiating with disrupted packing against the retinal chromophore at helices $C$ and $D$ before the unfolding transition, followed by small changes in helix $G$ near the site of retinal attachment both before and beyond the unfolding transition (Figure $2 \mathrm{c}$ ).

Chemical shift changes can be attributed to progressive changes in micelle-protein interactions with increasing SDS concentration and/or protein structural changes due to changes in $\mathrm{H}$-bonding and secondary structure during protein denaturation.

$\Delta \delta\left({ }^{1} \mathrm{H},{ }^{15} \mathrm{~N}\right)$ increased linearly with respect to $X_{\text {SDS }}$ at low $X_{\text {SDS }}$ (Figure 3a(i)) for 135 out of 190 residues $(71.1 \%)$ which were assignable up to $\geq 0.728 X_{\text {SDS }}(0.8 \%$ SDS in $0.5 \%$ c7-DHPC), as exemplified by Val-101. Most of these residues are distributed in the TM region (Figure $3 \mathrm{~b}$ ). This suggests that the chemical shift changes at low $X_{\text {SDS }}$ experienced by these backbone amides are attributable to gradual changes in micellar environment as SDS is being integrated into SDS/C7-DHPC mixed micelles. We propose that the slope of the linear correlation of $\Delta \delta\left({ }^{1} \mathrm{H},{ }^{15} \mathrm{~N}\right)$ against low $X_{\mathrm{SDS}}$ is a measure for the sensitivity of a given backbone amide towards changes in micellar environment. The distribution of different slopes across pSRII shows good consistency with $\Delta \delta\left({ }^{1} \mathrm{H},{ }^{15} \mathrm{~N}\right.$ ) (Figure 2a) for most helical residues (Figure S3), suggesting that (1) protein structural changes induced by SDS-mediated denaturation (which give rise to non-linearity in $\Delta \delta\left({ }^{1} \mathrm{H},{ }^{15} \mathrm{~N}\right)$ vs. $X_{\mathrm{SDS}}$ ) are small compared to progressive changes in chemical environment induced by changes in micelle composition (which give rise to linear correlations of $\Delta \delta\left({ }^{1} \mathrm{H},{ }^{15} \mathrm{~N}\right)$ vs. $X_{\mathrm{SDS}}$ in low $\left.X_{\mathrm{SDS}}\right)$, and/or (2) protein structural changes induced by SDS-mediated denaturation are localised to very few specific residues concentrating mostly at the edges of the helices.

Structural changes due to protein denaturation are expected to affect how backbone amides interact with the surrounding micellar environment. These are reflected as deviations from linearity in the linear correlation between $\Delta \delta\left({ }^{1} \mathrm{H},{ }^{15} \mathrm{~N}\right)$ and $X_{\mathrm{SDS}}$. The residuespecific point of denaturation is therefore assumed to be the lowest $X_{\text {SDS }}$ at which deviation from linearity arises in the $\Delta \delta\left({ }^{1} \mathrm{H},{ }^{15} \mathrm{~N}\right)$ vs. $X_{\mathrm{SDS}}$ graph. This allows the two main contributions towards chemical shift changes, namely changes in mixed micelle composition vs. protein structural changes due to protein denaturation, to be distinguished. Residues which became unobservable (see below) at lower $X_{\mathrm{SDS}}$ than the onset of non-linearity were omitted from this analysis. Residues deviating from linear $\Delta \delta\left({ }^{1} \mathrm{H},{ }^{15} \mathrm{~N}\right)$ vs. $X_{\mathrm{SDS}}$ relationships at $0.769 X_{\mathrm{SDS}}$ (1.0\% SDS in $0.5 \%$ c7-DHPC) mostly clustered at the extracellular half of helix C (Figure 3c). Other notable residues which showed early deviation from linearity included Tyr-51 on helix $\mathrm{B}$ and Asp-75 on helix $\mathrm{C}$, both of which are $\mathrm{H}$-bonded to the internal water network in the folded state, and Lys-205, to which the retinal is attached via a protonated Schiff base. This 
likely suggests that key inter-helical water-mediated $\mathrm{H}$-bonds and the retinal-binding pocket are beginning to be disrupted before the unfolding transition. Residues deviating from linear $\Delta \delta\left({ }^{1} \mathrm{H},{ }^{15} \mathrm{~N}\right)$ vs. $X_{\mathrm{SDS}}$ relationships starting from $0.834 X_{\mathrm{SDS}}(1.5 \%$ SDS in $0.5 \% \mathrm{c} 7-\mathrm{DHPC})$ were clustered in the middle portion of helix $D$. Residues showing the onset of a non-linear $\Delta \delta\left({ }^{1} \mathrm{H},{ }^{15} \mathrm{~N}\right)$ vs. $X_{S D S}$ relationship at even higher $X_{\text {SDS }}$ were mostly distributed at micelle/solvent interfaces or in solvent-accessible regions. Overall, these results suggest that protein structural changes due to SDS denaturation initiate at the extracellular half of helix $C$ and spread across to helix $D$ followed by residues at micelle interfaces and solventaccessible regions. This is consistent with the proposed sequence of structural changes in pSRII based on the aforementioned chemical shift analysis (see above).

19 residues (10.0\%) showed linear correlation of $\Delta \delta\left({ }^{1} \mathrm{H},{ }^{15} \mathrm{~N}\right)$ against the absolute concentration of SDS as exemplified by Leu-159 (Figure 3a(ii)), suggesting non-specific interaction of non-micellar SDS with these backbone amides. With the exceptions of Asn105 on helix D and Trp-171 on helix F, most of such residues were located in the loop regions ( 2 residues) and C-terminal tail ( 8 residues), or were located in the TM region and facing outwards toward the detergent micelle ( 7 residues) (Figure $3 b$ ).

There were 36 assignable residues which did not show obvious correlations against $X_{\text {SDS }}$ or the absolute concentration of SDS, suggesting complex changes in chemical environment with increasing SDS concentration. These residues were mostly distributed in the TM region of helices A, B and G, and faced the surrounding detergent environment (Figure 3b).

Tryptophan side-chain indoles which face the detergent micelle (Trp-9 and Trp-24 on helix A) experienced greater changes in chemical environment while Trp side-chains which face the protein core generally experienced little changes, consistent with the protein remaining embedded in a micellar environment and incorporation of SDS into the micelles (see Supplementary Information).

Altogether, the above detailed analysis of chemical shift changes provides insights into the heterogeneous nature of protein-micelle interactions and how such interactions change with increasing SDS concentrations and protein denaturation.

\section{pSRII shows increased conformational exchange in SDS-denatured states}

Line broadening could be caused by conformational exchange at the intermediate ( $\mu$ s-to-ms) exchange timescale [41] or slower molecular tumbling attributable to an increase in micelle size. Aggregation is unlikely to be the cause of peak broadening, because aggregation was shown to take more than $6 \mathrm{~h}$ at $35^{\circ} \mathrm{C}$ (308 K) (Figure S2; see Supplementary Information) and NMR experimental time was limited to $6 \mathrm{~h}$. The range of $X_{S D S}$ for which detailed analyses were made in this study $\left(0-0.870 X_{\text {SDS }}\right.$ 0-2.0\% SDS in $0.5 \% \mathrm{c7-DHPC}$ ) is below the reported concentration of SDS required for the formation of cylindrical micelles [45], and hence it is assumed that increase in micelle sizes and decrease in rotational correlation times are minor. It is also assumed that chemical exchange with water molecules is negligible due to the scarce presence of water molecules in the micellar environment and hydrophobicity of detergent acyl chains. 
Peak intensities generally decreased with increasing $X_{\text {SDS }}$ due to conformational exchange between different backbone amide conformations occurring at the intermediate ( $\mu$ s-to-ms) exchange timescale. This resulted in additional line broadening, as evidenced by most residues being no longer observable in $\geq 0.870 X_{\text {SDS }}$ (2.0\% SDS in 0.5\% c7-DHPC) (Figure 1a). Like in the folded state, residues in the C-terminal tail of pSRII remained significantly more flexible than the rest of the protein across all $X_{S D S}$, as evidenced by the strong intensities of these peaks. The large differences in peak intensities between TM vs. C-terminal residues indicate that the TM helices are likely to have remained embedded in SDS/C7-DHPC mixed micelles across all $X_{\text {SDS }}$ while there is little/no stable interaction of the C-terminal tail with detergent micelles, as previously mentioned. The very sharp and intense signals of the Cterminal residues are dominated by fast internal motions taking place on a ps-to-ns timescale. Such motions are highly sensitive to slight changes in viscosity and overall correlation time, as evidenced by the decrease in signal intensities of the C-terminal residues at high molar concentrations of SDS (> $100 \mathrm{mM}$ or $3.0 \%$ SDS [45]).

Short sample lifetimes (see Supplementary Information) and low experimental sensitivity limited the utilisation of NMR spin relaxation experiments. Hence, intensity ratios were used as proxy to report on increases in linewidth. The intensity ratio $\left(I / I_{0}\right)$ of each residue at different $X_{\text {SDS }}$ were obtained by expressing the normalised peak intensities as a ratio of the peak intensities at $0 X_{\text {SDS }}$. Larger $I / I_{0}$ represents the dominance of faster ps-to-ns local dynamics, whereas smaller $I / I_{0}$ indicates increased conformational fluctuations on the $\mu \mathrm{s}-$ to-ms timescale [46].

In the absence of SDS, the TM helices of pSRII are well-ordered, with some loop regions experiencing motions on the ps-to-ns timescale and a small number of residues in the protein core undergoing $\mu$ s-to-ms helix motions [39]. In the presence of SDS, most micelleembedded residues had $I / I_{0}<1$, which decreased with increasing $X_{\mathrm{SDS}}$ until the peaks became unobservable (Figure 4a), suggesting widespread transition from a well-ordered protein to one undergoing conformational fluctuations on the $\mu$ s-to-ms timescale. At 0.625 $X_{\mathrm{SDS}}$, most residues with $I / I_{0}>1$ were solvent-exposed or located at the micelle/solvent interface (Figure 4b), corresponding to increased fast-timescale motions and the lack of conformational exchange in the presence of SDS when compared to the folded state. Two residues within the retinal-binding pocket, namely Trp-76 in the middle of helix C and Val203 in the middle of helix $\mathrm{G}$, retained fast local dynamics $\left(I / I_{0}>1\right)$ and remained in the fast exchange regime in low $X_{\text {SDS. }}$ In $0.834 X_{\text {SDS }}$ (just above the unfolding transition), there was a marked decrease in the number of residues with $I / I_{0}>1$, indicating that pSRII denaturation is characterised by a general transition towards conformational fluctuations occurring on the $\mu s$-to-ms timescale, including residues in solvent-exposed regions and micelle/solvent interfaces. In $0.870 X_{\text {SDS }}$ in which pSRII is denatured (hydrolysed Schiff base), observable residues in the retinal-binding pocket were mostly clustered on helix $D$ and showed $I / I_{0}>1$ (Figure $4 \mathrm{~b}$ ). This indicates that the extracellular half of helix $\mathrm{D}$ exhibited a lack of $\mu$ s-to-ms timescale conformational fluctuations and showed faster local dynamics in high $X_{S D S}$ than in $0 X_{S D S}$, possibly suggesting that the extracellular half of helix $D$ becomes partially unfolded (helix unwound) during SDS denaturation. 
The number of observable residues decreased with increasing $X_{\text {SDS }}$, indicating augmented signal broadening due to conformational exchange with rates similar to the NMR timescale. Residues which were unassigned at 0.400 and $0.625 X_{S D S}$ were assumed to either be significantly overlapped with other peaks or intractable due to large chemical shift changes. Thr-80 on helix $\mathrm{C}$, which packs against $\mathrm{C} 9-\mathrm{C} 12$ at the middle of the retinal polyene chain in folded pSRII (Figure $5 a-b$ ), was the first residue that became unobservable starting from $0.769 X_{\text {SDS }}$. In $0.834 X_{\text {SDS }}$ (just above the unfolding transition), unobservable residues were concentrated amongst the cytoplasmic halves of helices $C, E, F$ and $G$, and between the extracellular halves of helices $A$ and $B$ and between helices $F$ and $G$. The chi-squared test (Table 2) shows that residues which became unobservable at $0.769 X_{\text {SDS }}$ and $0.834 X_{\text {SDS }}$ were significantly populated in helical regions $(P=0.026)$. Amongst such helical residues, there was significant preference for residues which face inwards towards the protein core $(P=$ 0.003 ) (Figure $5 c$ ). Unobservable residues within the retinal-binding pocket include Met-15 from helix A, Ala-47 from helix B, Thr-79 and Ile-83 from helix C, and Gly-207 and Phe-208 from helix $G$ (Figure $5 b$ ), all of which are located near the Schiff base, packing against Lys205 and up to C13-C14 of the retinal polyene chain. Peak disappearance is hypothesised to indicate loosening of inter-helical packing, which was initiated from the cytoplasmic side, likely amongst helices C, E, F and G, and propagated towards the Schiff base.

Residues which became unobservable at $0.870 X_{\text {SDS }}$ were spread across the entire protein (Figure 5a). The slight bias in the distribution of such residues toward the extracellular side could be because many residues in the cytoplasmic side have already disappeared at lower $X_{\text {SDS. }}$. The chi-squared test showed no significant difference in the distribution of unobservable residues between helical vs. loop residues or helical residues which face inwards (towards the protein core) vs. outwards (towards the detergent micelle) (Table 2 \& Figure 5c). Retinal-binding pocket residues which are within one helical turn away from the Schiff base, including Asp-201, Thr-204, Lys-205, Val-206 and Gly-209, became unobservable at $0.870 X_{S D S}$ (Figure $5 b$ ). This further supports the notion that residues surrounding the Schiff base were amongst residues which were disrupted at higher $X_{\text {SDS }}$ when the Schiff base is being hydrolysed.

Several residues which became unobservable at $0.834 X_{\text {SDS }}$ and $0.870 X_{\text {SDS, }}$ including Tyr-51 from helix B, and Asp-201 and Thr-204 in helix G, were H-bonded to internal water molecules in folded pSRII, suggesting that the water network was also being progressively disrupted with increasing $X_{S D S}$, consistent with our interpretation from chemical shift analysis.

In summary, pSRII denaturation was characterised by widespread increase in backbone amide conformational exchange on the $\mu s$-to-ms timescale, initiating from the cytoplasmic halves of helices $C, E, F$ and $G$ and propagating across the protein. Gradual disruption of the retinal-binding pocket began at $0.728 X_{\text {SDS }}$ with conformational exchange at Thr-80 which packed against the middle (C9-C12) of the retinal polyene chain, followed by several residues from helices $A, B, C$ and $G$ which packed against C13-C14 of retinal and Lys-205. The Schiff base and neighbouring residues in helix $\mathrm{G}$ were disrupted at higher $X_{\text {SDS }}$ compared to the rest of the retinal-binding pocket, as these residues in helix $\mathrm{G}$ showed prominent $\mu \mathrm{s}$ to-ms timescale conformational exchange at $\geq 0.870 X_{\text {SDS. }}$ In the denatured state $(\geq 0.870$ $X_{\text {SDS }}$ ), residues in helix $D$ were noted to show fast local dynamics while most residues 
showed pronounced conformational exchange at the $\mu$ s-to-ms timescale. The decrease in the number of observable residues highlights the inherent challenges in obtaining highresolution structural and dynamics information by NMR, especially for molecular processes with comparable timescales to the NMR timescale.

While most backbone amides experienced predominantly conformational exchange on the $\mu s$-to-ms timescale leading to line broadening (Figure S4a), the backbone amides of Val-17 and Phe-98 each had two resonances in 0.728-0.834 $X_{\text {SDS }}$ (Figure S4b), indicating slow exchange between two backbone conformations. A shift in the relative populations of these two conformations could be seen between 0.728 and $0.834 X_{\text {SDS. }}$ In $\geq 0.870 X_{\text {SDS }}$, all backbone amide peaks of Val-17 and Phe-98 showed decreased intensities, indicating a transition from slow exchange towards intermediate exchange as the peaks became increasingly broadened and eventually unobservable. From $0.909 X_{\text {SDS }}$ onwards, several other backbone amides were also found to undergo slow exchange between at least two conformations, as evidenced by difficulties in assigning several peaks in the glycine region of the spectrum due to big chemical shift changes (Figure S4c). While limited conclusions could be drawn due to the small number of residues involved and difficulties with resonance assignment, the above observations reflect different modes of backbone amide conformational exchange at different $X_{S D S}$, and highlight the heterogeneous nature of the ensemble of denatured states.

Decreased peak intensities were also observed with increasing $X_{\text {SDS }}$ for most tryptophan side-chain indoles, indicating that side-chains are also increasingly dominated by $\mu$-to-ms timescale conformational motions (see Supplementary Information).

\section{Acid-denatured pSRII shows disrupted water network and uncoupling of the helical bundle}

To compare the mechanisms of acid denaturation vs. SDS denaturation of pSRII, $2 \mathrm{D}\left[{ }^{1} \mathrm{H},{ }^{15} \mathrm{~N}\right]$ BEST-TROSY spectra were recorded for pSRII at progressively lower $\mathrm{pHs}(\mathrm{pH} 6.0, \mathrm{pH} 4.7, \mathrm{pH}$ 3.7, $\mathrm{pH}$ 2.6, $\mathrm{pH} 2.0$ ) in the presence of $0.5 \% \mathrm{c7}-\mathrm{DHPC}$ (Figure $6 \mathrm{a}) . \Delta \delta\left({ }^{1} \mathrm{H},{ }^{15} \mathrm{~N}\right)$ at acidic $\mathrm{pHs}$ were measured relative to chemical shifts at pH 6.0. Larger $\Delta \delta\left({ }^{1} \mathrm{H}^{15} \mathrm{~N}\right)$ were observed across all residues with decreasing pHs. $\Delta \delta\left({ }^{1} \mathrm{H},{ }^{15} \mathrm{~N}\right)$ were generally small, with $\Delta \delta\left({ }^{1} \mathrm{H},{ }^{15} \mathrm{~N}\right)<0.1 \mathrm{ppm}$ at $\mathrm{pH} 4.7$ and $\mathrm{pH} 3.7$ for most TM residues (Figure S5). Residues at the ends of helices consistently showed larger $\Delta \delta\left({ }^{1} \mathrm{H},{ }^{15} \mathrm{~N}\right)$ than TM residues. These results indicate that mildly acidic $\mathrm{pHs}$ led to only very small changes in $\mathrm{H}$-bonding and secondary structure, consistent with our previous biophysics studies showing no differences in helical content between folded pSRII at pH 6.0 and pSRII denatured at pH 2.0 [15]. Several residues spanning three helical turns at the cytoplasmic end of helix $D$ facing helix $E$ showed $\Delta \delta\left({ }^{1} \mathrm{H}^{15} \mathrm{~N}\right)>0.1 \mathrm{ppm}$ at $\mathrm{pH}$ 4.7, while residues in other helices with $\Delta \delta\left({ }^{1} \mathrm{H},{ }^{15} \mathrm{~N}\right)>0.1 \mathrm{ppm}$ were within one turn from helix ends (Figure S5a). Only two residues within the retinal-binding pocket had $\Delta \delta\left({ }^{1} \mathrm{H}^{15} \mathrm{~N}\right)>$ 0.1 ppm: Thr-80 on helix $\mathrm{C}(\mathrm{pH} 4.7$ and pH 3.7) and Met-15 on helix A (pH 3.7) (Figure S5b). This indicates that there is minimal disruption of the retinal-binding pocket and the tertiary structure of pSRII at $\mathrm{pH} 4.7$ and $\mathrm{pH} 3.7$, consistent with the samples remaining orange in colour after one week at room temperature (Figure $6 \mathrm{~b}$ ). 
Acid denaturation also led to increased conformational exchange on the $\mu$-to-ms timescale, as an increasing number of residues became broadened and unobservable (Figure $6 \mathrm{c}$ ). Residues which became unobservable at $\mathrm{pH} 4.7$ were located in the extracellular half, including Trp-76, Thr-79, Met-109 and Trp-178 within the retinal-binding pocket, an extensive stretch of helix C, and Val-206 and Gly-207 on helix G (Figure 6d). This suggests increased $\mu$ s-to-ms timescale conformational exchange around the Schiff base and within the retinal-binding pocket. At $\mathrm{pH} 3.7$, almost the entire helix $\mathrm{C}$ became unobservable, suggesting concerted changes in backbone dynamics across the entire helix. More residues within the retinal-binding pocket disappeared, including Ala-47 on helix B, Ile-83 on helix C, Gly-112 on helix D, Thr-204, Lys-205 and Phe-208 on helix G, indicating further loosening of the retinal-binding pocket and the Schiff base attachment site. It must be noted that many residues which became unobservable at $\mathrm{pH} 4.7$ or $\mathrm{pH} 3.7$ are $\mathrm{H}$-bonded to the internal water-mediated $\mathrm{H}$-bond network in the crystal structure of pSRII (PDB 1H68; [43]) (Figure $6 \mathrm{~d})$. This suggests that acid denaturation leads to disruption of the internal water-mediated $\mathrm{H}$-bond network. It can be inferred that the abolishment of key $\mathrm{H}$-bonds uncouples key residues from each other, thus enabling the backbone amides to sample different conformations on a $\mu$ s-to-ms timescale.

At $\mathrm{pH} 2.6$ and $\mathrm{pH} 2.0$, most TM residues became unobservable, indicating widespread $\mu$ s-toms timescale conformational exchange across the entire protein and suggesting that the TM helices are moving relative to each other as interhelical $\mathrm{H}$-bonds which hold the helical bundle together in the folded state become disrupted. Moreover, the samples appeared pale yellow in colour (Figure 6b), indicative of complete Schiff base hydrolysis and disruption of the retinal-binding pocket, and consistent with changes in the UV/vis absorption profile of the retinal chromophore and increase in tryptophan fluorescence intensity due to disruption of tertiary structure as reported in our previous studies [15]. Pronounced precipitation was observed after a week (Figure 6b), suggesting extensive exposure of aggregation-prone surfaces and/or decreased protein stability in the acid-denatured states.

In summary, acid denaturation of pSRII was characterised by disruption of the internal water network and uncoupling of helix $C$ from the rest of the helical bundle, as evidenced by $\mu$ s-toms timescale conformational exchange across almost the entire helix $C$, while chemical shift changes in the TM region remained very small.

\section{Discussion}

High-resolution NMR studies on full-length denatured membrane proteins are scarce in the literature. This likely reflects the challenges in studying a heterogeneous ensemble of denatured structures with different backbone dynamics and conformational exchange on different timescales. This paper presents detailed NMR studies on the structural and dynamics changes in different denatured states of pSRII by probing for changes in chemical shifts and peak intensities of backbone amides and tryptophan side-chain indoles (see Supplementary Information) under different denaturing conditions (SDS denaturation and acid denaturation).

\section{Structural and dynamics changes upon the loss of retinal during denaturation}


Under denaturing conditions in which the retinal chromophore gets liberated, SDS denaturation and acid denaturation both led to small chemical shift changes in the TM region (Figures $2 \&$ S5), indicative of little changes in secondary structure. Decreases in peak intensities were prominent across the seven TM helices (Figures 4-6), suggesting increased $\mu s$-to-ms timescale conformational exchange across pSRII backbone amides as a consequence of tertiary structure disruption.

It could therefore be inferred that the structure of the retinal-binding pocket and the dynamics of TM backbone amides are tightly linked to the presence of the all-trans retinal chromophore. This tight coupling is likely reflective of the close-fitted structural arrangements within the retinal-binding pocket in the folded state $[47,48]$, in which native retinal-protein interactions impose strong constraints on the backbone motions of the TM helices. As these constraints become relaxed and eventually abolished while the chromophore is being liberated during denaturation, the backbone amides become free to sample different conformations in the denatured states while at the same time remaining constrained within their $\alpha$-helical environments.

As demonstrated in our previous study, prolonged absence of all-trans retinal during SDS denaturation over the course of $72 \mathrm{~h}$ gradually reduces the refolding yield due to increase in aggregation and possible concomitant loss of the retinal-binding pocket conformation that is capable of rebinding retinal [15]. This suggests that different denatured conformations, some of which are prone to aggregation, readily exchange amongst each other, and that the energy barriers between these different conformations are small. However, the energy barrier for disaggregating denatured pSRII is large, making the aggregation of denatured pSRII de facto irreversible [15].

We assume that the retinal chromophore might influence the folding energy landscape by perturbing the equilibria amongst different backbone conformations of denatured pSRII or even the apo-protein state. Formation of the Schiff base via covalent linkage to Lys-205 and other native retinal-protein interactions during protein folding could, for example, stabilise particular conformations to favour folding and the generation of the native chromophore. Hypotheses on the possible influence of the retinal chromophore on protein folding has already emerged from studies on bovine rhodopsin by Sakamoto and Khorana [49]. The kinetics and the extent of retinal-binding during the regeneration of bacteriorhodopsin from bacterioopsin have also been studied using UV/vis spectroscopy [1,50-53], circular dichroism [50,54], tryptophan fluorescence $[51,53,55]$ and calorimetry [56]. Future studies on the structure and dynamics of the apo-protein are expected to yield more detailed insights on the roles of the retinal chromophore in the folding of pSRII and other retinalbinding proteins, and potentially open new avenues for investigating the importance of ligand-binding in the folding of other ligand-binding membrane proteins (e.g. GPCRs).

\section{Different unfolding pathways of pSRII}

There are notable differences between SDS- and acid-mediated denaturation, as evidenced by differences in the locations of structural changes and in the distribution of residues which 
became too broad for observation due to the presence of extensive $\mu$ s-to-ms exchange (Figure 7). The dependence of unfolding pathways on solvent conditions is presumably due to different retinal-protein interactions being disrupted and different denatured conformations being preferentially stabilised. That different solvent conditions give rise to different unfolding pathways has been shown previously in a study by Malmendal et al., where mapping the unfolding processes of bovine $\alpha$-lactalbumin in SDS, acid, GdmCl, TFE and heat by $1 \mathrm{D}{ }^{1} \mathrm{H}$ NMR combined with principal component analysis (GPS-NMR) showed that these denaturants move the protein away from the folded state in different directions [57], hence indicating different unfolding pathways. Further investigation into the properties of detergent micelles and high-resolution studies on protein-detergent interactions under different solvent conditions will also be valuable in future studies of denatured states and unfolding/folding pathways.

SDS denaturation of pSRII led to large chemical shift changes at the ends of helices, and progressive chemical shift changes around the retinal-binding pocket beginning from helices $C$ and $D$ before the unfolding transition, followed by small changes in helix $G$ near the site of retinal attachment beyond the unfolding transition (Figures $2 \& 7$ ). These chemical shift changes suggest that the retinal-binding pocket collapses gradually with increasing $X_{\text {SDS }}$, whereas Schiff base hydrolysis and loss of the retinal chromophore in high $X_{\text {SDS }}$ only leads to limited structural changes in helix $G$ and the rest of the helical bundle. While the ends of helices unravel, the helical content in the TM region is largely preserved.

In comparison, the secondary structure content is even more highly preserved in aciddenatured pSRII than in SDS-denatured pSRII, as evidenced by the small chemical shift changes observed under acidic conditions that were mostly restricted to the ends of helices (Figures $7 \&$ S5). This is consistent with the results of our previous CD studies, in which we found that there were no changes in alpha-helicity (MRE at $222 \mathrm{~nm}$ ) even after pSRII has been maintained at $\mathrm{pH} 2.0$ for $19 \mathrm{~h}$ [15].

The disruption of inter-helical interactions, assessed by a pronounced decrease in peak intensities or peak disappearance indicative of increased $\mu \mathrm{s}$-to-ms timescale conformational exchange, initiates from the cytoplasmic interface of helices $C, E, F$ and $G$ and spreads across the protein during SDS denaturation. The extracellular half of helix D shows faster local dynamics than in $0 X_{S D S}$, which suggests that this region is partially unfolded. This change in helix $D$ is reminiscent of the extracellular side of helix D in SDS-solubilised bacteriorhodopsin becoming partially accessible to bulk water due to denaturation, as assessed by laserinduced oxidative labelling and mass spectrometry [58].

In acid denaturation, helix $\mathrm{C}$ is the first helix to be uncoupled from the helical bundle, with peaks disappearing across almost the entire helix $\mathrm{C}$ at $\mathrm{pH}$ 3.7. Several residues which are $\mathrm{H}$-bonded via the internal water network also exhibited $\mu$ s-to-ms timescale motion in aciddenatured pSRII (Figures 6-7), thus further supporting the disruption of the retinal-binding pocket and the internal water network. Interestingly, the above observations are strongly reminiscent of the loss of connectivity between helices $C$ and $G$ in the light-activated states of pSRII, in which movements (relative to the ground state) of the side-chains of Asp-75 and Lys-205 in the early $\mathrm{K}$ intermediate [59] and in the signalling $M$ intermediate [60] provide a mechanism for the disordering and displacement of the water molecule Wat-402. Wat-402 
is located between and H-bonded to the Schiff base (Lys-205), Asp-75 and Asp-201 in the ground state structure [43]. These structural changes lead to the abolishment of key $\mathrm{H}$ bonds with the central water cluster, thereby uncoupling helices $C$ and $G$ from each other during light activation.

Acidic $\mathrm{pH}$ leads to protonation of ionisable groups and changes in electrostatic interactions. Residues which have ionisable side-chains and are located near/within the retinal-binding pocket include the Schiff base counterion Asp-75 ( $\mathrm{pKa}=3.4$ in phosphatidylcholinereconstituted wild-type pSRII [61] and DDM-solubilised pSRII [62,63]), Asp-193 (pKa = 6.4 [64]) and Asp-201 (pKa = 1.2 [63]). Taking into account the $\mathrm{pH}$ range explored and the changes in peak intensities observed in this study, it is evident that progressive lowering of the $\mathrm{pH}$ from 6.0 to 2.0 leads to the protonation of Asp-75.

Protonation of Asp-75 is hypothesised to lead to the disruption of the H-bond network bridging Wat-402, the Schiff base (Lys-205), Asp-75 and Asp-201 [43]. Changes in dynamics occur as this $\mathrm{H}$-bond network becomes progressively disrupted at decreasing $\mathrm{pHs}$, as evidenced by the backbone amide of Asp-75 becoming unobservable at $\mathrm{pH} \mathrm{4.7,} \mathrm{that} \mathrm{of} \mathrm{Lys-}$ 205 becoming unobservable at $\mathrm{pH} 3.7$ while that of Asp-201 remains observable at both $\mathrm{pH}$ 4.7 and $\mathrm{pH}$ 3.7. On the other hand, local structural changes at backbone amides are very small, as evidenced by the small chemical shift changes ( $<0.05 \mathrm{ppm}$ ) experienced by Asp201 and Lys-205. The lack of structural and dynamics changes at Asp-201 in mildly acidic pHs is consistent with its low $\mathrm{pKa}$ and concomitant lack of change in protonation state during the $\mathrm{pH}$ titration. While the proton release group Asp-193 is also $\mathrm{H}$-bonded to the water network, its high $\mathrm{pKa}$ suggests that this residue may already be partially protonated at $\mathrm{pH}$ 6.0, and is unlikely to be the main driver behind the changes observed during acid denaturation.

\section{Summary}

Using solution-state NMR, we have studied the denaturation of pSRII using SDS and acidic $\mathrm{pH}$. This paper presents one of the very few [29] detailed backbone NMR studies on a fulllength, denatured membrane protein, and has shed insights on differences in the unfolding pathways under different denaturing conditions. SDS denaturation leads to fraying of helix ends, small structural changes within the binding pocket, and $\mu$ s-to-ms timescale conformational exchange that initiates from the cytoplasmic side of helices $C, E, F$ and $G$ and propagates across to most of the 7TM helices except for the extracellular half of helix $D$, which shows fast local dynamics that could suggest at least partial unfolding. Acid denaturation leads to fraying of helix ends but otherwise very limited structural changes, and $\mu$ s-to-ms timescale conformational exchange that initiates from helix $\mathrm{C}$ and propagates across the internal water-mediated $\mathrm{H}$-bond network and all 7TM helices. Based on the widespread $\mu$ s-to-ms timescale conformational exchange in pSRII backbone amides under the denaturing conditions explored (high $X_{\mathrm{SDS}}$ and acidic $\mathrm{pHs}$ ), it is further proposed that the all-trans retinal chromophore might influence the folding energy landscape by perturbing the equilibria amongst different conformations sampled by the backbone amides in the absence of native retinal-protein interactions. 
Once again, we emphasise pSRII as a model protein which is highly suitable for membrane protein folding studies. It is hoped that the high-resolution molecular insights on the denatured states of pSRII presented in this paper could pave the way for furthering our understanding on the folding and unfolding pathways of other retinal-binding proteins and membrane proteins in general, and also for investigating the importance of ligand-binding in the folding pathways of other ligand-binding membrane proteins, such as GPCRs.

\section{Materials and Methods}

\section{Materials}

SDS (electrophoresis grade) and 50\% (w/v) hydroxylamine hydrochloride were purchased from Sigma-Aldrich. Diheptanoylphosphatidylcholine (c7-DHPC) was purchased from Anatrace.

\section{Protein expression and purification}

Natronomonas pharaonis sensory rhodopsin II (pSRII) was expressed in E. coli Tuner(DE3)Lacl cells (Novagen) and purified in $50 \mathrm{mM}$ sodium phosphate pH 6.0, $50 \mathrm{mM}$ $\mathrm{NaCl}, 0.05 \%(\mathrm{w} / \mathrm{v})$ sodium azide $\left(\mathrm{NaN}_{3}\right)$ and $2 \%(\mathrm{w} / \mathrm{v}) \mathrm{c7}-\mathrm{DHPC}$ as described previously [40]. Protein concentration was measured using a molar extinction coefficient of $48000 \mathrm{M}^{-1} \mathrm{~cm}^{-1}$ for the native retinal chromophore absorbing at $498 \mathrm{~nm}$, or $49390 \mathrm{M}^{-1} \mathrm{~cm}^{-1}$ for absorbance at $280 \mathrm{~nm}[65]$.

\section{D ${ }^{1} \mathrm{H}$ NMR}

1D ${ }^{1} \mathrm{H}$ NMR spectra were measured using a Bruker Avancelll AV800 equipped with $5 \mathrm{~mm}$ TXI CryoProbe $(\mathrm{HCN} / \mathrm{z})$. Pulse sequences were 1D spin echos. Water suppression was achieved using a 3-9-19 $180^{\circ}$ WATERGATE sequence. All spectra were recorded at $308 \mathrm{~K}$, with 512 complex data points (51.2 ms), 512 scans, and a spectral width of $10000 \mathrm{~Hz}$. Selective suppression of detergent signals was achieved using a Gaussian Q5 cascade with duration of $5 \mathrm{~ms}$ followed by purging by gradient pulses.

\section{D ${ }^{1} \mathrm{H},{ }^{15} \mathrm{~N}$ NMR}

All 2D $\left[{ }^{1} \mathrm{H},{ }^{15} \mathrm{~N}\right]$ BEST-TROSY experiments were recorded with samples of ${ }^{15} \mathrm{~N}$-labelled pSRII in $50 \mathrm{mM} \mathrm{NaH} \mathrm{PO}_{4} / \mathrm{Na}_{2} \mathrm{HPO}_{4}, 50 \mathrm{mM} \mathrm{NaCl}, 0.5 \%$ c7-DHPC with varied $\mathrm{pH}$ and SDS concentrations, at $308 \mathrm{~K}$. All spectra were recorded on a Bruker Avancelll AV800 spectrometer equipped with a $5 \mathrm{~mm}$ TXI CryoProbe $(\mathrm{HCN} / \mathrm{z})$, with $512 \times 128$ complex points, spectral width of $2777.78 \mathrm{~Hz}$ in the indirect dimension ( $t_{1 \max }=46.08 \mathrm{~ms}$ ) and acquisition time of $51.2 \mathrm{~ms}$. Frequency discrimination in the ${ }^{15} \mathrm{~N}$ dimension was achieved using $\mathrm{P} / \mathrm{N}$ type coherence order selection combined with axial peak shifting by one-half the spectral width. Solvent suppression was not required due to the utilisation of amide-selective ${ }^{1} \mathrm{H}$ pulses for magnetisation transfer.

Details of the sample concentrations and the number of scans for each reported spectrum are shown in Table 3.

\section{Data analysis}

Chemical shift differences were calculated as: 


$$
\Delta \delta\left({ }^{1} \mathrm{H},{ }^{15} \mathrm{~N}\right)=\sqrt{\Delta \delta\left({ }^{1} \mathrm{H}\right)^{2}+\frac{\Delta \delta\left({ }^{15} \mathrm{~N}\right)^{2}}{6}}
$$

Intensity ratios were calculated as:

$$
I / I_{0} \text { (intensity ratio) }=\frac{\text { Peak intensity at } n X_{\mathrm{SDS}}}{\text { Peak intensity at } 0 X_{\mathrm{SDS}}}
$$

\section{SDS-PAGE}

SDS-PAGE analysis of pSRII at different SDS concentrations was performed on a $12 \%$ polyacrylamide gel at $90 \mathrm{~V}$ for $15 \mathrm{~min}$ followed by $180 \mathrm{~V}$ for $40 \mathrm{~min}$. $2 \times$ Native loading dye containing $62.5 \mathrm{mM}$ Tris- $\mathrm{HCl}, \mathrm{pH} 6.8,25 \%$ (v/v) glycerol, $0.01 \%$ (w/v) bromophenol blue [66] was used for the analysis of aggregation states in denatured protein samples. PageRuler prestained protein ladder (10-180 kDa) (Thermo Fisher Scientific) was used as molecular weight markers. Protein bands were visualised using the Pierce ${ }^{\mathrm{TM}}$ Silver Stain Kit (Thermo Fisher Scientific) according to the manufacturer's instructions.

\section{Far-UV CD spectroscopy}

To avoid strong UV absorbance by $\mathrm{NaN}_{3}, \mathrm{NaN}_{3}$ was first removed from small aliquots (100 $\left.\mu \mathrm{l}\right)$ of pSRII stock solutions by concentrating and diluting 3 times in a $0.5 \mathrm{ml}$ centrifugal filter unit (10-kDa cutoff) (Merck Millipore) using a total of $\sim 1.5 \mathrm{ml}$ of $50 \mathrm{mM}$ sodium phosphate $\mathrm{pH}$ 6.0, $50 \mathrm{mM} \mathrm{NaCl}, 0.1 \%(\mathrm{w} / \mathrm{v}) \mathrm{c7}-\mathrm{DHPC}$. CD spectra were recorded at $25^{\circ} \mathrm{C}$ by scanning between 194 and $250 \mathrm{~nm}$ on an Aviv 410 spectrometer (Aviv Biomedical Inc.) using a $1 \times 1$ $\mathrm{mm}$ cuvette. Each sample contains $\sim 0.17 \mathrm{mg} / \mathrm{ml}$ protein in a volume of $400 \mu \mathrm{l}$, measured $2 \mathrm{~h}$ after exposure to unfolding buffer. $C D$ spectra of the buffer solutions were subtracted from the sample spectra and smoothed using a window width of 11 and a degree of 2. Spectra were reported as the average of 3 scans. Data for each experimental condition were reported as the average of 3 independent samples.

Molar residue ellipticity (MRE; $[\theta]$ ) was calculated as:

$$
[\theta]=\frac{\theta}{10 \times l \times c \times n}
$$

where $\theta$ is the observed ellipticity (millidegrees), $l$ the path length $(\mathrm{cm}), c$ the protein concentration (M) and $n$ the number of peptide bonds, taken as 246 for pSRII.

\section{Calculations}

The mole fraction of SDS ( $\left.X_{\text {SDS }}\right)$ was calculated as the bulk detergent composition in solution.

$$
X_{S D S}=\frac{[\mathrm{SDS}]}{[\mathrm{SDS}]+[\mathrm{c} 7-\mathrm{DHPC}]}
$$




\section{References}

[1] K.S. Huang, H. Bayley, M.J. Liao, E. London, H.G. Khorana, Refolding of an integral membrane protein. Denaturation, renaturation, and reconstitution of intact bacteriorhodopsin and two proteolytic fragments., J. Biol. Chem. 256 (1981) 38023809.

[2] M.J. Liao, E. London, H.G. Khorana, Regeneration of the native bacteriorhodopsin structure from two chymotryptic fragments, J Biol Chem. 258 (1983) 9949-9955.

[3] J.L. Popot, S.E. Gerchman, D.M. Engelman, Refolding of bacteriorhodopsin in lipid bilayers. A thermodynamically controlled two-stage process, J. Mol. Biol. 198 (1987) 655-676. doi:10.1016/0022-2836(87)90208-7.

[4] J.L. Popot, D.M. Engelman, Membrane protein folding and oligomerization: the twostage model, Biochemistry. 29 (1990) 4031-4037. doi:10.1021/bi00469a001.

[5] H. Sigrist, R.H. Wenger, E. Kislig, M. Wüthrich, Refolding of bacteriorhodopsin: Protease V8 fragmentation and chromophore reconstitution from proteolytic V8 fragments, Eur. J. Biochem. 177 (1988) 125-133. doi:10.1111/j.14321033.1988.tb14352.x.

[6] T.W. Kahn, D.M. Engelman, Bacteriorhodopsin can be refolded from two independently stable transmembrane helices and the complementary five-helix fragment, Biochemistry. 31 (1992) 6144-6151. doi:10.1021/bi00141a027.

[7] S. Ozawa, R. Hayashi, A. Masuda, T. lio, S. Takahashi, Reconstitution of bacteriorhodopsin from a mixture of a proteinase V8 fragment and two synthetic peptides, Biochim. Biophys. Acta - Biomembr. 1323 (1997) 145-153. doi:10.1016/S0005-2736(96)00182-4.

[8] T. Marti, Refolding of bacteriorhodopsin from expressed polypeptide fragments, J. Biol. Chem. 273 (1998) 9312-9322. doi:10.1074/jbc.273.15.9312.

[9] L.J. Catoire, M. Zoonens, C. Van Heijenoort, F. Giusti, É. Guittet, J.L. Popot, Solution NMR mapping of water-accessible residues in the transmembrane $\beta$-barrel of OmpX, Eur. Biophys. J. 39 (2010) 623-630.

[10] K.D. Ridge, S.S. Lee, L.L. Yao, In vivo assembly of rhodopsin from expressed polypeptide fragments., Proc. Natl. Acad. Sci. U. S. A. 92 (1995) 3204-3208. doi:10.1073/pnas.92.8.3204.

[11] K.D. Ridge, S.S.J. Lee, N.G. Abdulaev, Examining rhodopsin folding and assembly through expression of polypeptide fragments, J. Biol. Chem. 271 (1996) 7860-7867. doi:10.1074/jbc.271.13.7860.

[12] K.D. Ridge, T. Ngo, S.S.J. Lee, N.G. Abdulaev, Folding and assembly in rhodopsin. Effect of mutations in the sixth transmembrane helix on the conformation of the third cytoplasmic loop, J. Biol. Chem. 274 (1999) 21437-21442. doi:10.1074/jbc.274.30.21437.

[13] K.D. Ridge, N.G. Abdulaev, Folding and assembly of rhodopsin from expressed fragments, Methods Enzymol. 315 (2000) 59-70. doi:10.1016/S0076-6879(00)158343.

[14] J. Klein-Seetharaman, Dual role of interactions between membranous and soluble portions of helical membrane receptors for folding and signaling, Trends Pharmacol. Sci. 26 (2005) 183-189. doi:10.1016/j.tips.2005.02.009.

[15] Y.L. Tan, J. Mitchell, J. Klein-Seetharaman, D. Nietlispach, Characterization of Denatured States and Reversible Unfolding of Sensory Rhodopsin II, J. Mol. Biol. 430 (2018) 4068-4086. doi:10.1016/j.jmb.2018.07.031. 
[16] O. Tastan, E. Yu, M. Ganapathiraju, A. Aref, A.J. Rader, J. Klein-Seetharaman, Comparison of stability predictions and simulated unfolding of rhodopsin structures., Photochem. Photobiol. 83 (2007) 351-362. doi:10.1562/2006-06-20-RA-942.

[17] P. Curnow, P.J. Booth, Combined kinetic and thermodynamic analysis of $\alpha$-helical membrane protein unfolding, Proc. Natl. Acad. Sci. U. S. A. 104 (2007) 18970-18975. doi:10.1073/pnas.0705067104.

[18] J.P. Schlebach, Z. Cao, J.U. Bowie, C. Park, Revisiting the folding kinetics of bacteriorhodopsin, Protein Sci. 21 (2012) 97-106. doi:10.1002/pro.766.

[19] O. Tastan, A. Dutta, P. Booth, J. Klein-Seetharaman, Retinal proteins as model systems for membrane protein folding, Biochim. Biophys. Acta - Bioenerg. 1837 (2014) 656663. doi:10.1016/j.bbabio.2013.11.021.

[20] D.M. Korzhnev, L.E. Kay, Probing invisible, low-populated states of protein molecules by relaxation dispersion NMR spectroscopy: An application to protein folding, Acc. Chem. Res. 41 (2008) 442-451. doi:10.1021/ar700189y.

[21] M. Kovermann, P. Rogne, M. Wolf-Watz, Protein dynamics and function from solution state NMR spectroscopy, Q. Rev. Biophys. 49 (2016) e6. doi:10.1017/S0033583516000019.

[22] H.J. Dyson, P.E. Wright, Insights into protein folding from NMR, Annu. Rev. Phys. Chem. 47 (1996) 369-395. doi:10.1146/annurev.physchem.47.1.369.

[23] M. Zeeb, J. Balbach, Protein folding studied by real-time NMR spectroscopy, Methods. 34 (2004) 65-74. doi:10.1016/j.ymeth.2004.03.014.

[24] K.H. Mok, P.J. Hore, Photo-CIDNP NMR methods for studying protein folding, Methods. 34 (2004) 75-87. doi:10.1016/j.ymeth.2004.03.006.

[25] P. Neudecker, P. Lundström, L.E. Kay, Relaxation dispersion NMR spectroscopy as a tool for detailed studies of protein folding, Biophys. J. 96 (2009) 2045-2054. doi:10.1016/j.bpj.2008.12.3907.

[26] P. Neudecker, P. Robustelli, A. Cavalli, P. Walsh, P. Lundström, A. Zarrine-Afsar, S. Sharpe, M. Vendruscolo, L.E. Kay, Structure of an intermediate state in protein folding and aggregation, Science (80-. ). 336 (2012) 362-366. doi:10.1126/science.1214203.

[27] C.A. Waudby, H. Launay, L.D. Cabrita, J. Christodoulou, Protein folding on the ribosome studied using NMR spectroscopy, Prog. Nucl. Magn. Reson. Spectrosc. 74 (2013) 57-75. doi:10.1016/j.pnmrs.2013.07.003.

[28] A. Zhuravleva, D.M. Korzhnev, Protein folding by NMR, Prog. Nucl. Magn. Reson. Spectrosc. 100 (2017) 52-77. doi:10.1016/j.pnmrs.2016.10.002.

[29] T. Jacso, B. Bardiaux, J. Broecker, S. Fiedler, T. Baerwinkel, A. Mainz, U. Fink, C. Vargas, H. Oschkinat, S. Keller, B. Reif, The mechanism of denaturation and the unfolded state of the $\alpha$-helical membrane-associated protein Mistic, J. Am. Chem. Soc. 135 (2013) 18884-18891. doi:10.1021/ja408644f.

[30] S. Tuzi, S. Yamaguchi, A. Naito, R. Needleman, J.K. Lanyi, H. Saito, Conformation and dynamics of $\left[3-{ }^{13} \mathrm{C}\right] \mathrm{Ala}$ - labeled bacteriorhodopsin and bacterioopsin, induced by interaction with retinal and its analogs, as studied by ${ }^{13} \mathrm{C}$ nuclear magnetic resonance, Biochemistry. 35 (1996) 7520-7527. doi:10.1021/bi960274s.

[31] J. Klein-Seetharaman, P.J. Reeves, M.C. Loewen, E.V. Getmanova, J. Chung, H. Schwalbe, P.E. Wright, H.G. Khorana, Solution NMR spectroscopy of $\left[\alpha-{ }^{15} \mathrm{~N}\right]$ lysinelabeled rhodopsin: The single peak observed in both conventional and TROSY-type HSQC spectra is ascribed to Lys-339 in the carboxyl-terminal peptide sequence, Proc. Natl. Acad. Sci. U. S. A. 99 (2002) 3452-3457. doi:10.1073/pnas.052713999. 
[32] J. Klein-Seetharaman, N.V.K. Yanamala, F. Javeed, P.J. Reeves, E.V. Getmanova, M.C. Loewen, H. Schwalbe, H.G. Khorana, Differential dynamics in the G protein-coupled receptor rhodopsin revealed by solution NMR., Proc. Natl. Acad. Sci. U. S. A. 101 (2004) 3409-3413. doi:10.1073/pnas.0308713101.

[33] A. Dutta, C. Altenbach, S. Mangahas, N. Yanamala, E. Gardner, W.L. Hubbell, J. KleinSeetharaman, Differential dynamics of extracellular and cytoplasmic domains in denatured states of rhodopsin, Biochemistry. 53 (2014) 7160-7169. doi:10.1021/bi401557e.

[34] K. V. Pervushin, V.Y. Orekhov, A.I. Popov, L.Y. Musina, A.S. Arseniev, Threedimensional structure of (1-71)bacterioopsin solubilized in methanol/chloroform and SDS micelles determined by ${ }^{15} \mathrm{~N}-{ }^{1} \mathrm{H}$ heteronuclear NMR spectroscopy, Eur. J. Biochem. 219 (1994) 571-583. doi:10.1111/j.1432-1033.1994.tb19973.x.

[35] V.Y. Orekhov, K. V. Pervushin, D.M. Korzhnev, A.S. Arseniev, Backbone dynamics of (1-71)- and (1-36)bacterioopsin studied by two-dimensional ${ }^{1} \mathrm{H}-{ }^{15} \mathrm{~N} N \mathrm{NMR}$ spectroscopy, J. Biomol. NMR. 6 (1995) 113-122. doi:10.1007/BF00211774.

[36] M. Poms, P. Ansorge, L. Martinez-Gil, S. Jurt, D. Gottstein, K.E. Fracchiolla, L.S. Cohen, P. Guntert, I. Mingarro, F. Naider, O. Zerbe, NMR Investigation of Structures of Gprotein Coupled Receptor Folding Intermediates., J. Biol. Chem. 291 (2016) 2717027186. doi:10.1074/jbc.M116.740985.

[37] N.E. Zhou, B.Y. Zhu, B.D. Sykes, R.S. Hodges, Relationship between amide proton chemical shifts and hydrogen bonding in amphipathic $\alpha$-helical peptides, J. Am. Chem. Soc. 114 (1992) 4320-4326. doi:10.1021/ja00037a042.

[38] S.P. Mielke, V. V. Krishnan, Characterization of protein secondary structure from NMR chemical shifts, Prog. Nucl. Magn. Reson. Spectrosc. 54 (2009) 141-165. doi:10.1016/j.pnmrs.2008.06.002.

[39] A. Gautier, J.P. Kirkpatrick, D. Nietlispach, Solution-state NMR spectroscopy of a seven-helix transmembrane protein receptor: Backbone assignment, secondary structure, and dynamics, Angew. Chemie - Int. Ed. 47 (2008) 7297-7300. doi:10.1002/anie.200802783.

[40] A. Gautier, H.R. Mott, M.J. Bostock, J.P. Kirkpatrick, D. Nietlispach, Structure determination of the seven-helix transmembrane receptor sensory rhodopsin II by solution NMR spectroscopy., Nat. Struct. Mol. Biol. 17 (2010) 768-774. doi:10.1038/nsmb.1807.

[41] M.P. Williamson, Using chemical shift perturbation to characterise ligand binding, Prog. Nucl. Magn. Reson. Spectrosc. 73 (2013) 1-16. doi:10.1016/j.pnmrs.2013.02.001.

[42] C.A. Waudby, A. Ramos, L.D. Cabrita, J. Christodoulou, Two-dimensional NMR lineshape analysis, Sci. Rep. 6 (2016) 24826. doi:10.1038/srep24826.

[43] A. Royant, P. Nollert, K. Edman, R. Neutze, E.M. Landau, E. Pebay-Peyroula, J. Navarro, X-ray structure of sensory rhodopsin II at 2.1-Å resolution, Proc. Nat. Acad. Sci. USA. 98 (2001) 10131-10136. doi:10.1073/pnas.181203898.

[44] E. Pebay-Peyroula, A. Royant, E.M. Landau, J. Navarro, Structural basis for sensory rhodopsin function, Biochim. Biophys. Acta - Biomembr. 1565 (2002) 196-205. doi:10.1016/S0005-2736(02)00569-2.

[45] Y. Croonen, E. Gelade, M. Van Der Zegel, H. M. Van der Auweraer, Vandendriessche, F.C. De Schryver, M. Almgren, Influence of salt, detergent concentration, and temperature on the fluorescence quenching of 1-methylpyrene in sodium dodecyl 
sulfate with $m$-dicyanobenzene, J. Phys. Chem. 87 (1983) 1426-1431.

[46] A.G. Palmer, NMR characterization of the dynamics of biomacromolecules, Chem. Rev. 104 (2004) 3623-3640. doi:10.1021/cr030413t.

[47] J. Hirayama, Y. Imamoto, Y. Shichida, T. Yoshizawa, A.E. Asato, R.S.H. Liu, N. Kamo, Shape of the chromophore binding site in pharaonis phoborhodopsin from a study using retinal analogs, Photochem. Photobiol. 60 (1994) 388-393. doi:10.1111/j.17511097.1994.tb05121.x.

[48] J. Hirayma, N. Kamo, Y. Imamoto, Y. Shichida, T.^ru Yoshizawa, Reason for the lack of light-dark adaptation in pharaonis phoborhodopsin: reconstitution with 13-cis-retinal, FEBS Lett. 364 (1995) 168-170. doi:10.1016/0014-5793(95)00381-I.

[49] T. Sakamoto, H.G. Khorana, Structure and function in rhodopsin: the fate of opsin formed upon the decay of light-activated metarhodopsin II in vitro, Proc. Natl. Acad. Sci. U. S. A. 92 (1995) 249-253. doi:10.1073/pnas.92.1.249.

[50] E. London, H.G. Khorana, Denaturation and renaturation of bacteriorhodopsin in detergents and lipid-detergent mixtures., J. Biol. Chem. 257 (1982) 7003-7011.

[51] P.J. Booth, A. Farooq, S.L. Flitsch, Retinal binding during folding and assembly of the membrane protein bacteriorhodopsin, Biochemistry. 35 (1996) 5902-5909. doi:10.1021/bi960129e.

[52] P.J. Booth, A. Farooq, Intermediates in the assembly of bacteriorhodopsin investigated by time-resolved absorption spectroscopy, Eur. J. Biochem. 246 (1997) 674-680. doi:10.1111/j.1432-1033.1997.00674.x.

[53] P.J. Booth, M.L. Riley, S.L. Flitsch, R.H. Templer, A. Farooq, A.R. Curran, N. Chadborn, P. Wright, Evidence that bilayer bending rigidity affects membrane protein folding, Biochemistry. 36 (1997) 197-203. doi:10.1021/bi962200m.

[54] M.L. Riley, B.A. Wallace, S.L. Flitsch, P.J. Booth, Slow $\alpha$ helix formation during folding of a membrane protein, Biochemistry. 36 (1997) 192-196. doi:10.1021/bi962199r.

[55] P.J. Booth, S.L. Flitsch, L.J. Stern, D.A. Greenhalgh, P.S. Kim, H.G. Khorana, Intermediates in the folding of the membrane protein bacteriorhodopsin, Nat. Struct. Biol. 2 (1995) 139-143. doi:10.1038/nsb0295-139.

[56] T.W. Kahn, D.M. Engelman, J.M. Sturtevant, Thermodynamic measurements of the contributions of helix-connecting loops and of retinal to the stability of bacteriorhodopsin, Biochemistry. 31 (1992) 8829-8839. doi:10.1021/bi00152a020.

[57] A. Malmendal, J. Underhaug, D.E. Otzen, N.C. Nielsen, Fast mapping of global protein folding states by multivariate NMR: A GPS for proteins, PLoS One. 5 (2010) e10262. doi:10.1371/journal.pone.0010262.

[58] Y. Pan, L. Brown, L. Konermann, Mapping the structure of an integral membrane protein under semi-denaturing conditions by laser-induced oxidative labeling and mass spectrometry, J. Mol. Biol. 394 (2009) 968-981. doi:10.1016/j.jmb.2009.09.063.

[59] K. Edman, A. Royant, P. Nollert, C.A. Maxwell, E. Pebay-Peyroula, J. Navarro, R. Neutze, E.M. Landau, Early structural rearrangements in the photocycle of an integral membrane sensory receptor, Structure. 10 (2002) 473-482. doi:10.1016/S09692126(02)00736-0.

[60] A. Ishchenko, E. Round, V. Borshchevskiy, S. Grudinin, I. Gushchin, J.P. Klare, A. Remeeva, V. Polovinkin, P. Utrobin, T. Balandin, M. Engelhard, G. Büldt, V. Gordeliy, New insights on signal propagation by sensory rhodopsin II/transducer complex, Sci. Rep. 7 (2017) 41811. doi:10.1038/srep41811.

[61] M. Iwamoto, Y. Sudo, K. Shimono, T. Araiso, N. Kamo, Correlation of the O- 
intermediate rate with the pKa of Asp-75 in the dark, the counterion of the Schiff base of pharaonis phoborhodopsin (sensory rhodopsin II), Biophys. J. 88 (2005) 12151223. doi:10.1529/biophysj.104.045583.

[62] I. Chizhov, G. Schmies, R. Seidel, J.R. Sydor, B. Luttenberg, M. Engelhard, The photophobic receptor from Natronobacterium pharaonis: temperature and $\mathrm{pH}$ dependencies of the photocycle of sensory rhodopsin II, Biophys. J. 75 (1998) 9991009. doi:10.1016/s0006-3495(98)77588-5.

[63] K. Shimono, M. Kitami, M. Iwamoto, N. Kamo, Involvement of two groups in reversal of the bathochromic shift of pharaonis phoborhodopsin by chloride at low $\mathrm{pH}$, Biophys. Chem. 87 (2000) 225-230. doi:10.1016/S0301-4622(00)00195-2.

[64] M. Iwamoto, C. Hasegawa, Y. Sudo, K. Shimono, T. Araiso, N. Kamo, Proton release and uptake of pharaonis phoborhodopsin (sensory rhodopsin II) reconstituted into phospholipids, Biochemistry. 43 (2004) 3195-3203. doi:10.1021/bi035960n.

[65] B. Scharf, B. Hess, M. Engelhard, Chromophore of sensory rhodopsin II from Halobacterium halobium., Biochemistry. 31 (1992) 12486-92. doi:10.1021/bi00065a045.

[66] A. Dutta, K.C. Tirupula, U. Alexiev, J. Klein-Seetharaman, Characterization of membrane protein non-native states. 1. Extent of unfolding and aggregation of rhodopsin in the presence of chemical denaturants, Biochemistry. 49 (2010) 63176328. doi:10.1021/bi100338e. 
Table 1:

\begin{tabular}{|c|c|}
\hline Helix & Residue \\
\hline \multirow{2}{*}{ A } & Met-15 \\
\hline & Phe-22 \\
\hline \multirow{5}{*}{ B } & Leu-40 \\
\hline & Ile-43 \\
\hline & Ser-44 \\
\hline & Ile-46 \\
\hline & Ala-47 \\
\hline \multirow{5}{*}{ C } & Tyr-73 \\
\hline & Trp-76 \\
\hline & Thr-79 \\
\hline & Thr-80 \\
\hline & Ile-83 \\
\hline \multirow{4}{*}{ D } & Val-108 \\
\hline & Met-109 \\
\hline & Ala-111 \\
\hline & Gly-112 \\
\hline \multirow{4}{*}{$E$} & Phe-127 \\
\hline & Gly-130 \\
\hline & Ala-131 \\
\hline & Phe-134 \\
\hline \multirow{4}{*}{$\mathrm{F}$} & Trp-171 \\
\hline & Tyr-174 \\
\hline & Pro-175 \\
\hline & Trp-178 \\
\hline \multirow{9}{*}{ G } & Asp-201 \\
\hline & Leu-202 \\
\hline & Val-203 \\
\hline & Thr-204 \\
\hline & Lys-205 \\
\hline & Val-206 \\
\hline & Gly-207 \\
\hline & Phe-208 \\
\hline & Gly-209 \\
\hline
\end{tabular}

Residues in the retinal-binding pocket, identified by taking a $3 \AA$ cut-off around the retinal chromophore 
Table 2:

\begin{tabular}{|l|c|c|}
\hline & $\mathbf{0 . 7 6 9} \boldsymbol{\&} \mathbf{0 . 8 3 4} \boldsymbol{X}_{\text {SDS }}$ & $\mathbf{0 . 8 7 0} \boldsymbol{X}_{\text {SDs }}$ \\
\hline Helical vs. Loop & $P=0.026\left({ }^{*}\right)$ & $P=0.093$ \\
\hline $\begin{array}{l}\text { Inward-facing vs. Outward facing } \\
\text { (helical residues only) }\end{array}$ & $P=0.003\left({ }^{* *}\right)$ & $P=0.481$ \\
\hline
\end{tabular}

$P$-values from chi-squared test on the distribution of residues which have become unobservable at different $X_{\text {SDS }}$

Table 3:

\begin{tabular}{|c|c|c|c|c|c|}
\hline Figure & $X_{\text {SDS }}(\%$ SDS) & [c7-DHPC] (\%) & $\mathrm{pH}$ & [pSRII] $(\mu \mathrm{M})$ & No. of Scans \\
\hline \multirow{2}{*}{ Fig S1 } & $0.769(1.0 \%)$ & 0.5 & \multirow{2}{*}{6.0} & \multirow{2}{*}{94} & \multirow{2}{*}{240} \\
\hline & $0.769(2.0 \%)$ & 0.9 & & & \\
\hline \multirow{12}{*}{$\begin{array}{l}\text { Figs 1-5, } \\
\text { S2-4, S6 }\end{array}$} & 0 & \multirow{12}{*}{0.5} & \multirow{12}{*}{6.0} & 83 & 160 \\
\hline & $0.400(0.2 \%)$ & & & 94 & \multirow{11}{*}{240} \\
\hline & $0.625(0.5 \%)$ & & & 94 & \\
\hline & $0.728(0.8 \%)$ & & & 94 & \\
\hline & $0.769(1.0 \%)$ & & & 94 & \\
\hline & $0.834(1.5 \%)$ & & & 83 & \\
\hline & $0.870(2.0 \%)$ & & & 94 & \\
\hline & $0.893(2.5 \%)$ & & & 94 & \\
\hline & 0.909 (3.0\%) & & & 83 & \\
\hline & $0.971(10 \%)$ & & & 83 & \\
\hline & $0.985(20 \%)$ & & & 83 & \\
\hline & $0.990(30 \%)$ & & & 83 & \\
\hline \multirow{4}{*}{ Figs 6 , S5 } & \multirow{4}{*}{0} & \multirow{4}{*}{0.5} & 4.7 & \multirow{4}{*}{112} & \multirow{4}{*}{240} \\
\hline & & & 3.7 & & \\
\hline & & & 2.6 & & \\
\hline & & & 2.0 & & \\
\hline
\end{tabular}

Data acquisition parameters for NMR experiments on ${ }^{15} \mathrm{~N}$-pSRII in different $X_{\text {SDS }}$ and at different pHs

All samples contained $10 \% \mathrm{D}_{2} \mathrm{O}$ and TSP as internal chemical shift reference. All spectra were recorded at $308 \mathrm{~K}$. Signal intensities were scaled according to number of scans and protein concentration prior to analysis. 


\section{Figure legends}

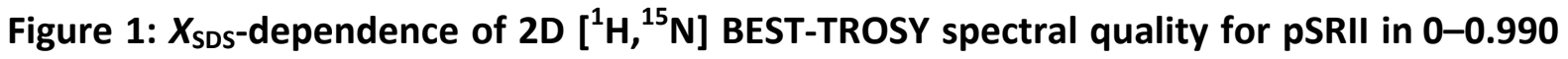
$X_{\text {SDS }}(0-30 \%$ SDS in $0.5 \%$ c7-DHPC)

All spectra were recorded with $\sim 90 \mu \mathrm{M}{ }^{15} \mathrm{~N}$-labelled pSRII, on an $800 \mathrm{MHz}$ spectrometer at $308 \mathrm{~K}$, with 240 scans. All samples contained TSP as internal chemical shift reference. (a) Overlay of 2D [ $\left.{ }^{1} \mathrm{H},{ }^{15} \mathrm{~N}\right]$ BEST-TROSY spectra of pSRII in 0 (black), 0.625 (red), 0.870 (teal), 0.971 (blue) and 0.990 (magenta) $X_{\text {SDS }}(0 \%, 0.5 \%, 2.0 \%, 10 \%$ and $30 \%$ SDS in $0.5 \%$ c7-DHPC).

(b) Expanded view showing peak shifts of the glycine residues (boxed region in panel a) in 0$0.990 X_{\text {SDS }}(0-30 \%$ SDS in 0.5\% c7-DHPC). Chemical shift changes are represented using arrows next to each peak. (c) Example of non-linear changes in resonance positions for Ser44 in $0-0.909 X_{S D S}\left(0-3 \%\right.$ SDS in 0.5\% c7-DHPC). Resonances in different $X_{\text {SDS }}$ are coloured according to the colour scheme in panel b. (d) Backbone amides with non-linear changes in resonance positions are represented as blue spheres on the solution-state NMR structure of pSRII (PDB 2KSY), showing widespread conformational exchange across the protein.

Figure 2: Chemical shift changes of pSRII backbone amides at different $X_{\text {SDS, }}$ suggesting fraying of helix ends and progressive disruption of the retinal-binding pocket $\Delta \delta\left({ }^{1} \mathrm{H},{ }^{15} \mathrm{~N}\right)$ are mapped onto the solution-state NMR structure of pSRII (PDB 2KSY). (a) Residues are coloured from blue to red according to $\Delta \delta\left({ }^{1} \mathrm{H},{ }^{15} \mathrm{~N}\right)$ values, showing greater structural changes at the ends of helices than around the retinal-binding pocket and suggesting fraying of helix ends with increasing $X_{\text {SDs. }}$. Unassigned residues are coloured white. (b) Surface representation (wired mesh) of the retinal-binding pocket (residues within $3 \AA$ of retinal) coloured according to $\Delta \delta\left({ }^{1} \mathrm{H},{ }^{15} \mathrm{~N}\right)$ values. Residues with $\Delta \delta\left({ }^{1} \mathrm{H},{ }^{15} \mathrm{~N}\right)>0.1 \mathrm{ppm}$ are shown using stick representation, suggesting progressive disruption of the retinal-binding pocket. (c) Extracellular view of pSRII. Retinal-binding pocket residues with $\Delta \delta\left({ }^{1} \mathrm{H},{ }^{15} \mathrm{~N}\right)>0.1$ ppm at $0.769 X_{\text {SDS }}$ are shown using stick representation.

Figure 3: Linear correlation of $\Delta \delta\left({ }^{1} \mathrm{H},{ }^{15} \mathrm{~N}\right)$ against $X_{\mathrm{SDS}}$ and the absolute concentration of SDS

(a) Examples of linear relationships (i) between $\Delta \delta\left({ }^{1} \mathrm{H},{ }^{15} \mathrm{~N}\right)$ of $\mathrm{Val}-101$ and $X_{\mathrm{SDS}}$, and (ii) between $\Delta \delta\left({ }^{1} \mathrm{H},{ }^{15} \mathrm{~N}\right)$ of Leu-159 and the absolute concentration of SDS ([SDS]) in \%. The fitted equations and goodness-of-fit $\left(R^{2}\right)$ of the linear regressions are shown in the bottom right corners of (i) and (ii). In (i), Val-101 shows deviation from linearity of $\Delta \delta\left({ }^{1} \mathrm{H},{ }^{15} \mathrm{~N}\right)$ against $X_{\mathrm{SDS}}$ from $0.870 X_{\mathrm{SDS}}(2 \%$ SDS in $0.5 \% \mathrm{c} 7-\mathrm{DHPC})$ onwards, suggesting that protein denaturation leads to changes in how the backbone amide of Val-101 interacts with surrounding micellar SDS. In (ii), Leu-159 shows linear relationship between $\Delta \delta\left({ }^{1} \mathrm{H},{ }^{15} \mathrm{~N}\right)$ and [SDS] up to $2 \%$ SDS in $0.5 \% \mathrm{c} 7-\mathrm{DHPC}\left(0.870 \mathrm{X}_{\mathrm{SDS}}\right)$, beyond which the signal intensity drops below the noise level. (b) Residues are coloured according to their correlations against $X_{\text {SDS }}$ or [SDS]. (c) Residues are coloured according to the range of $X_{\text {SDS }}$ in which a linear relationship between $\Delta \delta\left({ }^{1} \mathrm{H},{ }^{15} \mathrm{~N}\right)$ and $X_{\text {SDS }}$ was observed.

\section{Figure 4: Intensity ratios at different $X_{\text {SDS }}$}

(a) Residues with $I / I_{0}>1$ are shown as lime spheres, and residues with $I / I_{0}<1$ are coloured purple. Solvent-exposed regions of folded pSRII in $0 X_{S D S}$, determined from NMR signal attenuation in the presence of the soluble spin label reagent gadoteridol [40], are shaded in pale blue. (b) Extracellular view of pSRII, highlighting the observation of residues 
in the extracellular half of helix D in the SDS-denatured state $\left(0.870 X_{\text {SDS }} ; 2.0 \%\right.$ SDS in $0.5 \%$ c7-DHPC). Residues are coloured according to $I / I_{0}$ values, the retinal chromophore and residues with $I / I_{0}>1$ are shown as sticks, and the retinal-binding pocket is represented as wired mesh.

\section{Figure 5: Resonances undergoing pronounced loss of intensity at different $\boldsymbol{X}_{\text {SDS }}$}

(a) Residues with intensities that drop below the noise level at $0.769,0.834$ and $0.870 X_{\text {SDS }}$ are shown as red, yellow and cyan spheres, respectively. Residues which remain observable at $\geq 0.893 X_{\text {SDS }}$ are coloured in blue. (b) Surface representation (wired mesh) of the retinalbinding pocket (residues within $3 \AA$ of retinal) coloured according to $X_{\mathrm{SDS}}$ at which the backbone amide resonance becomes unobservable due to excessive broadening, and residues which become unobservable at 0.769-0.870 $X_{\text {SDS }}$ are shown using stick representation. (c) Extracellular and cytoplasmic views of pSRII, showing a greater proportion of inward-facing helical residues amongst the residues that experience prominent intensity losses at 0.769 and $0.834 X_{\text {SDS }}$.

\section{Figure 6: Acid denaturation of pSRII}

(a) 2D $\left[{ }^{1} \mathrm{H},{ }^{15} \mathrm{~N}\right]$ BEST-TROSY spectra of pSRII at different acidic pHs. All spectra were recorded at $800 \mathrm{MHz}\left({ }^{1} \mathrm{H}\right)$ at $308 \mathrm{~K}$ with 240 scans. All samples contained $\sim 90 \mu \mathrm{M}{ }^{15} \mathrm{~N}-\mathrm{pSRII}$ and TSP as internal chemical shift reference. (b) Photos of $\sim 90 \mu \mathrm{M}{ }^{15} \mathrm{~N}$-pSRII at different acidic $\mathrm{pHs}$ after one week at room temperature. (c) Residues which became unobservable at $\mathrm{pH} 4.7$ and $\mathrm{pH} 3.7$ are shown as red and yellow spheres, respectively. Residues which became unobservable at $\mathrm{pH} 2.6$ are coloured cyan. Residues which remained observable at $\mathrm{pH} 2.0$ are coloured blue. (d) Residues which are $\mathrm{H}$-bonded to the internal water-mediated $\mathrm{H}$-bond network are shown as sticks on the crystal structure of pSRII (PDB 1H68) and coloured according to the $\mathrm{pH}$ at which the residue became unobservable due to prominent loss of signal intensity. The $\mathrm{H}$-bonds in the internal water network are shown as grey dashed lines. Water molecules are shown as spheres, with internal water molecules coloured blue.

\section{Figure 7. Summary models for SDS and acid denaturation of pSRII}

Folded pSRII in c7-DHPC at pH 6.0 has seven well-ordered transmembrane helices (blue rods) and an all-trans retinal chromophore (black sticks) that is covalently attached to helix $\mathrm{G}$ via a Schiff base linkage to Lys-205. SDS denaturation leads to slight unwinding of helix ends (blue-to-white gradients at the ends of helices), but otherwise small changes in secondary structure. In low $X_{\text {SDS }}$ where the retinal chromophore remains present, the cytoplasmic side of helices $C, E, F$ and $G$ undergo $\mu$ s-to-ms timescale conformational exchange (red colour), and structural changes can be detected within the binding pocket (cyan colour). Above $0.834 X_{\mathrm{SDS}}$, Schiff base hydrolysis occurs, the retinal chromophore is lost, and most of the 7TM helices undergo $\mu$ s-to-ms timescale conformational exchange except for the extracellular half of helix $D$, which shows fast local dynamics (lime colour). Acid denaturation leads to very minimal changes in secondary structure. In mildly acidic pHs, the retinal chromophore remains present and most of helix $C$ experiences $\mu$ s-to-ms timescale conformational exchange. At pH 2.6 and below, Schiff base hydrolysis occurs, the retinal chromophore is lost, and all 7TM helices undergo $\mu$ s-to-ms timescale conformational exchange. 
(a)

$0.625 X_{\text {SDS }}$
$(0.5 \%$ SDS $)$

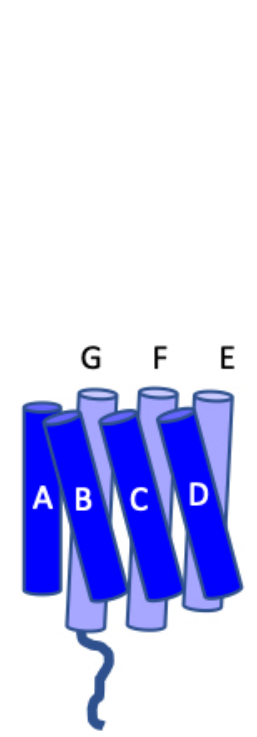

$\overbrace{}^{180^{\circ}}$

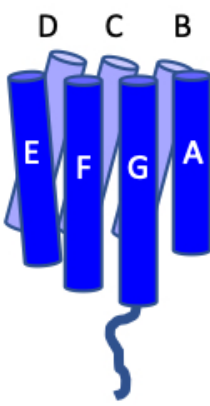

(b)

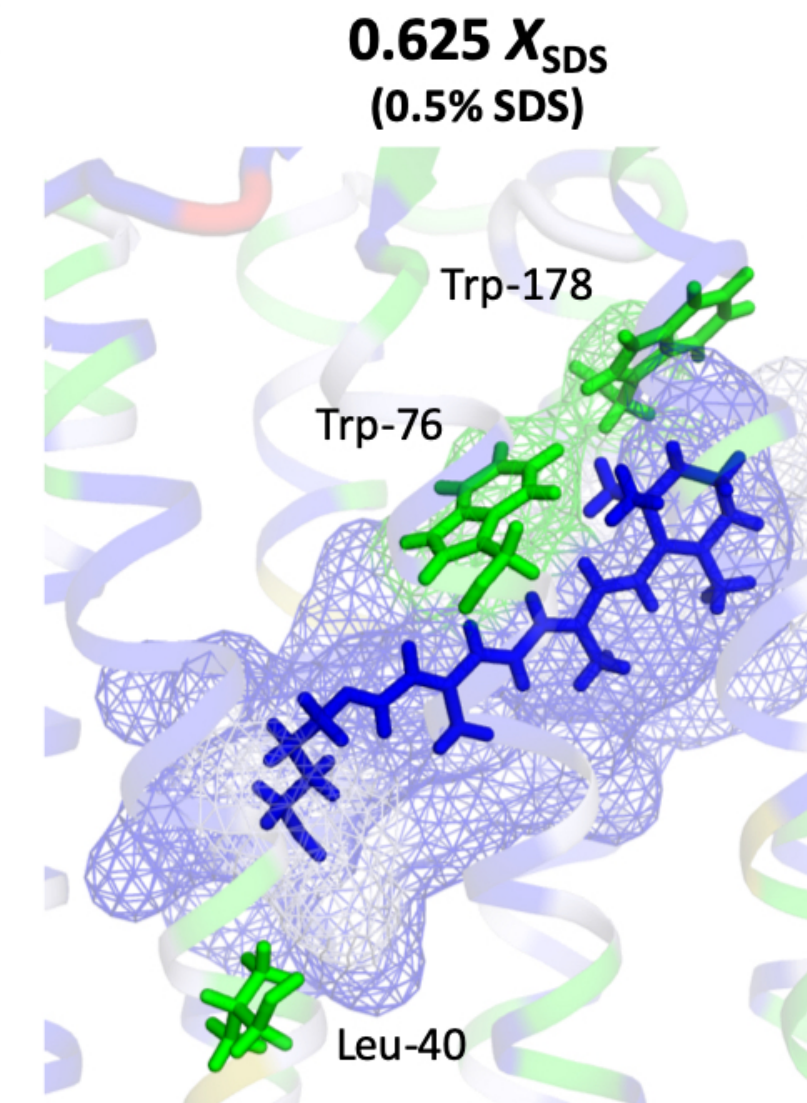

$\Delta \delta\left({ }^{1} \mathrm{H},{ }^{15} \mathrm{~N}\right)$
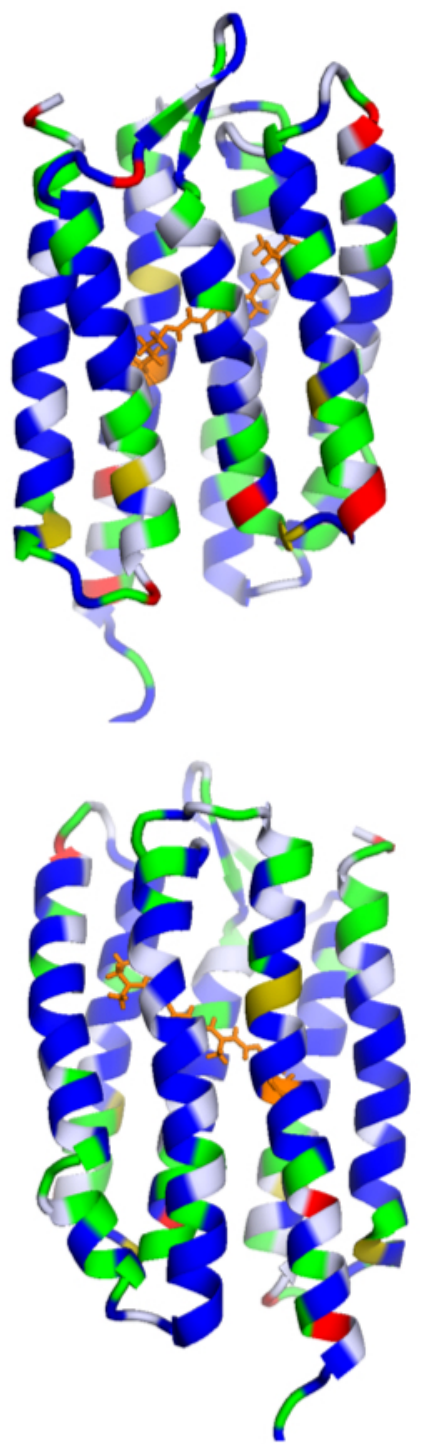

$0.728 X_{\text {SDS }}$
$(0.8 \%$ SDS $)$

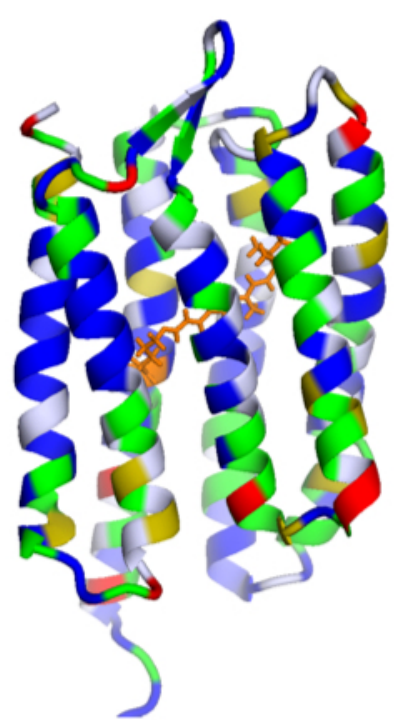

$\Delta \delta\left({ }^{1} \mathrm{H},{ }^{15} \mathrm{~N}\right)$
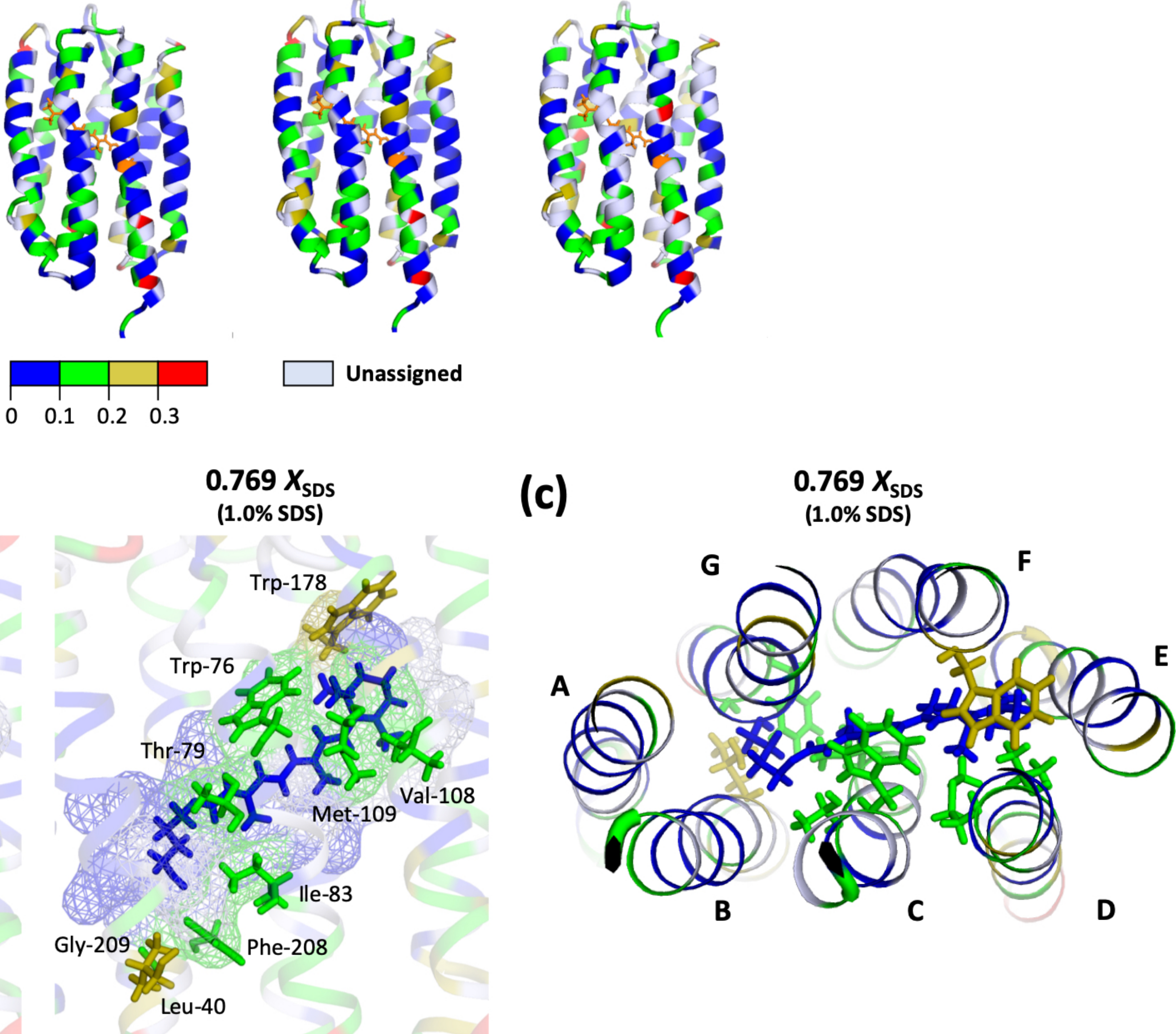

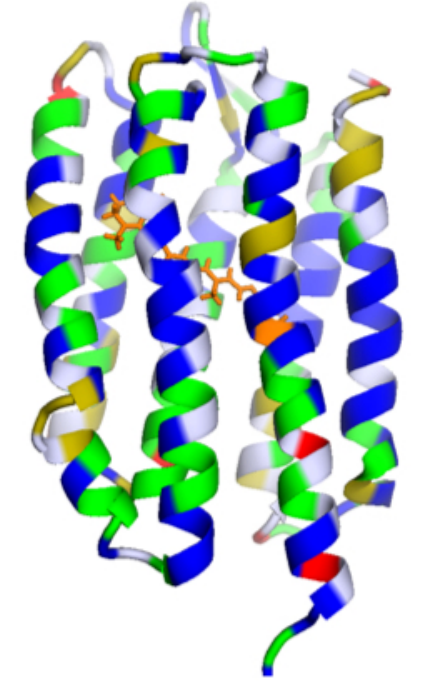

Unassigned

(c)

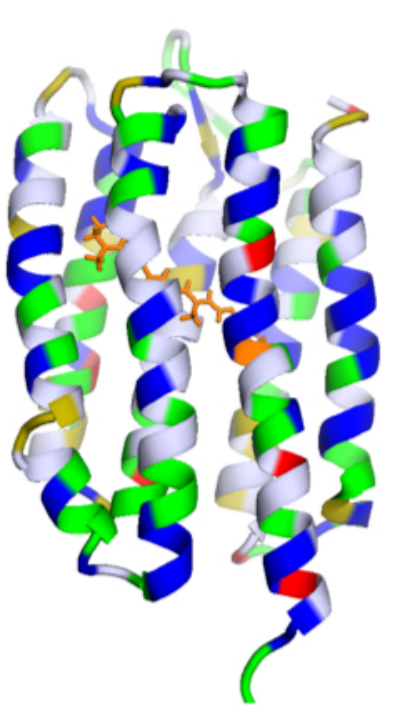

$0.834 X_{\text {SDS }}$

(1.5\% SDS)

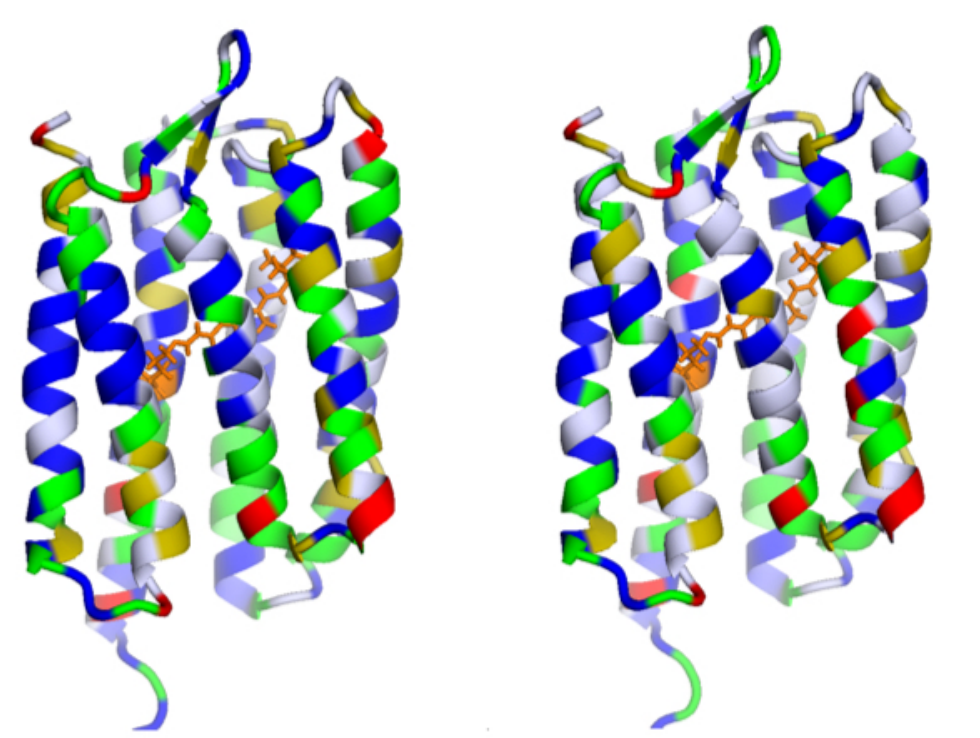

C
D 

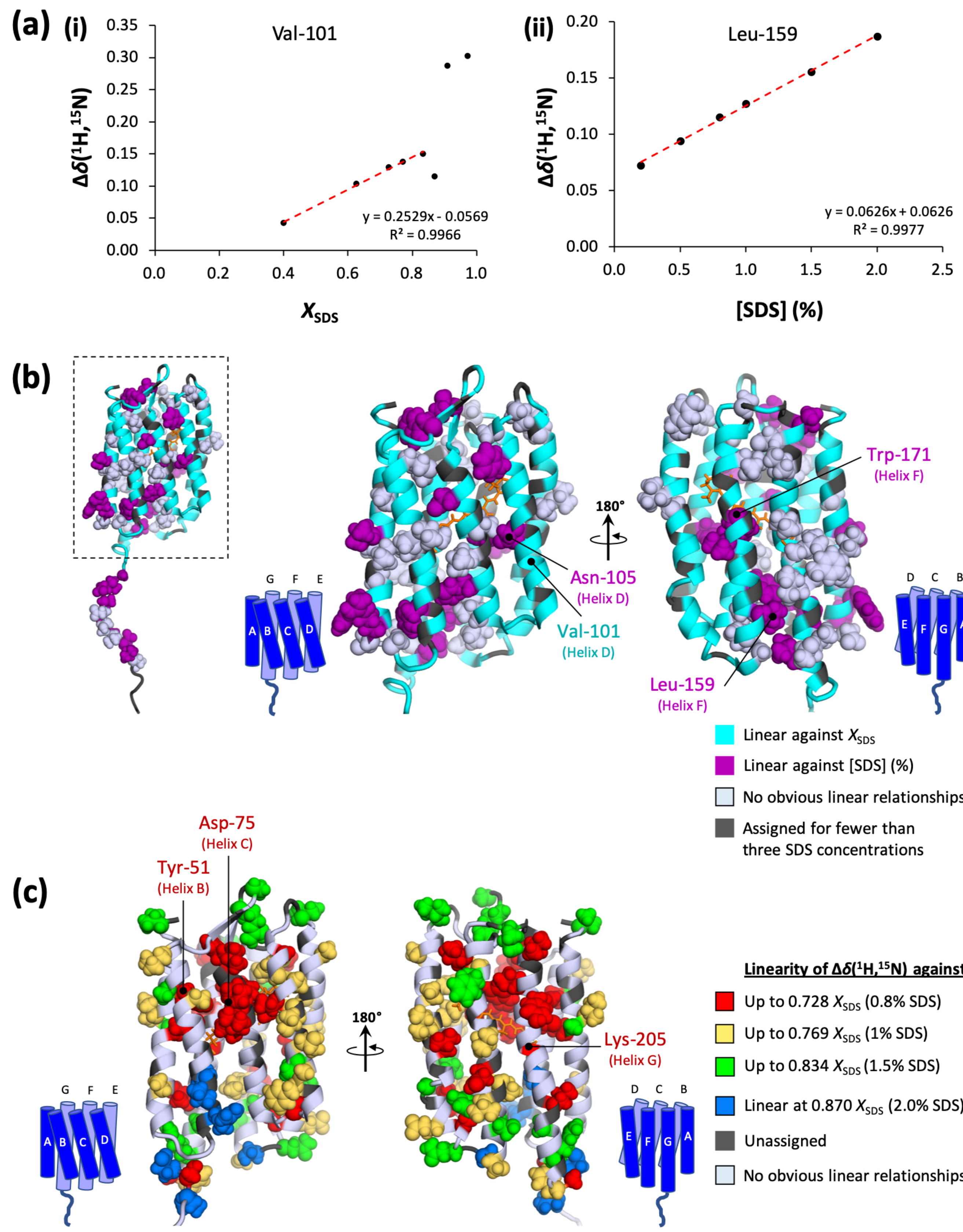

\section{Linearity of $\Delta \delta\left({ }^{1} \mathrm{H}^{15} \mathrm{~N}\right)$ against $X_{S D S}$}

$\square$ Up to $0.728 X_{\text {SDS }}(0.8 \%$ SDS)

Up to $0.769 X_{\text {SDS }}(1 \% S D S)$

Up to $0.834 X_{\text {SDS }}(1.5 \%$ SDS $)$

Linear at $0.870 X_{\mathrm{SDS}}(2.0 \% \mathrm{SDS})$

Unassigned

No obvious linear relationships 


\section{$0.625 X_{\mathrm{SDS}}$ \\ (0.5\% SDS)}

(a)
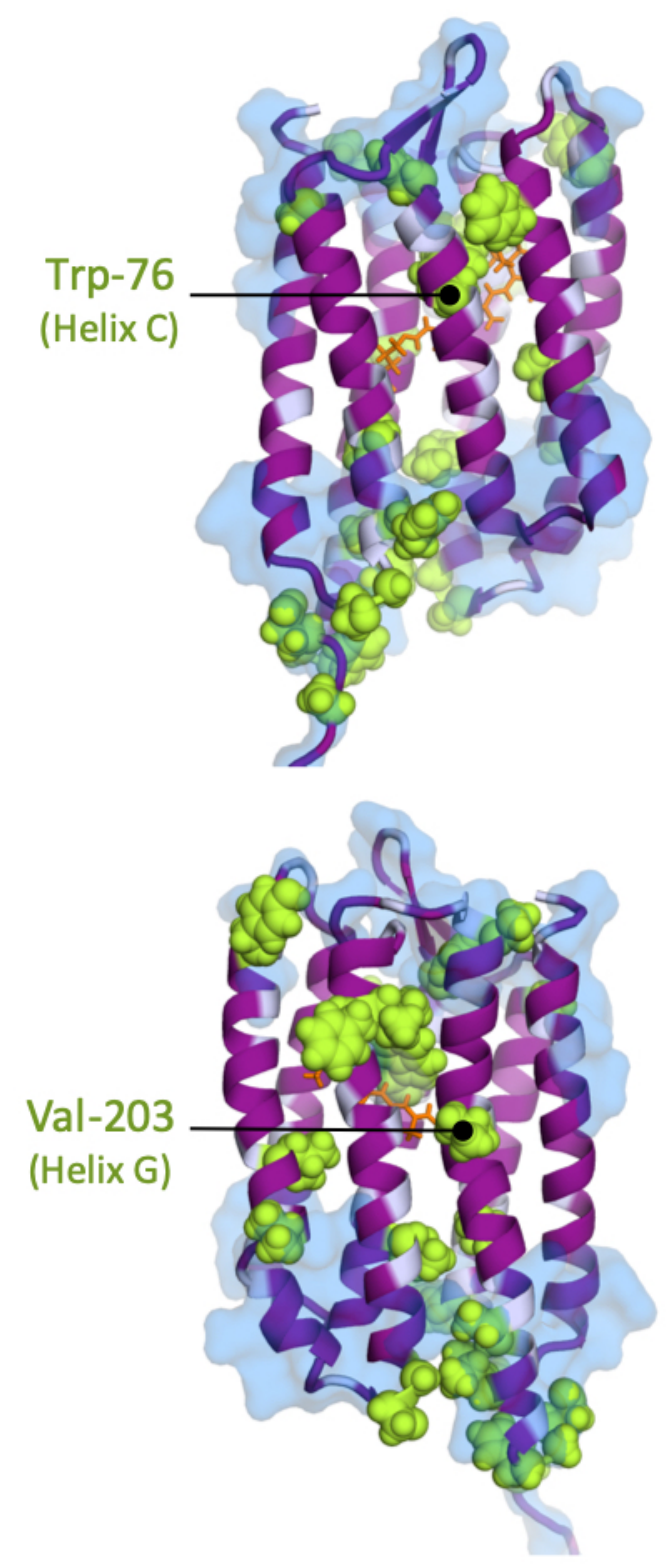

(b)

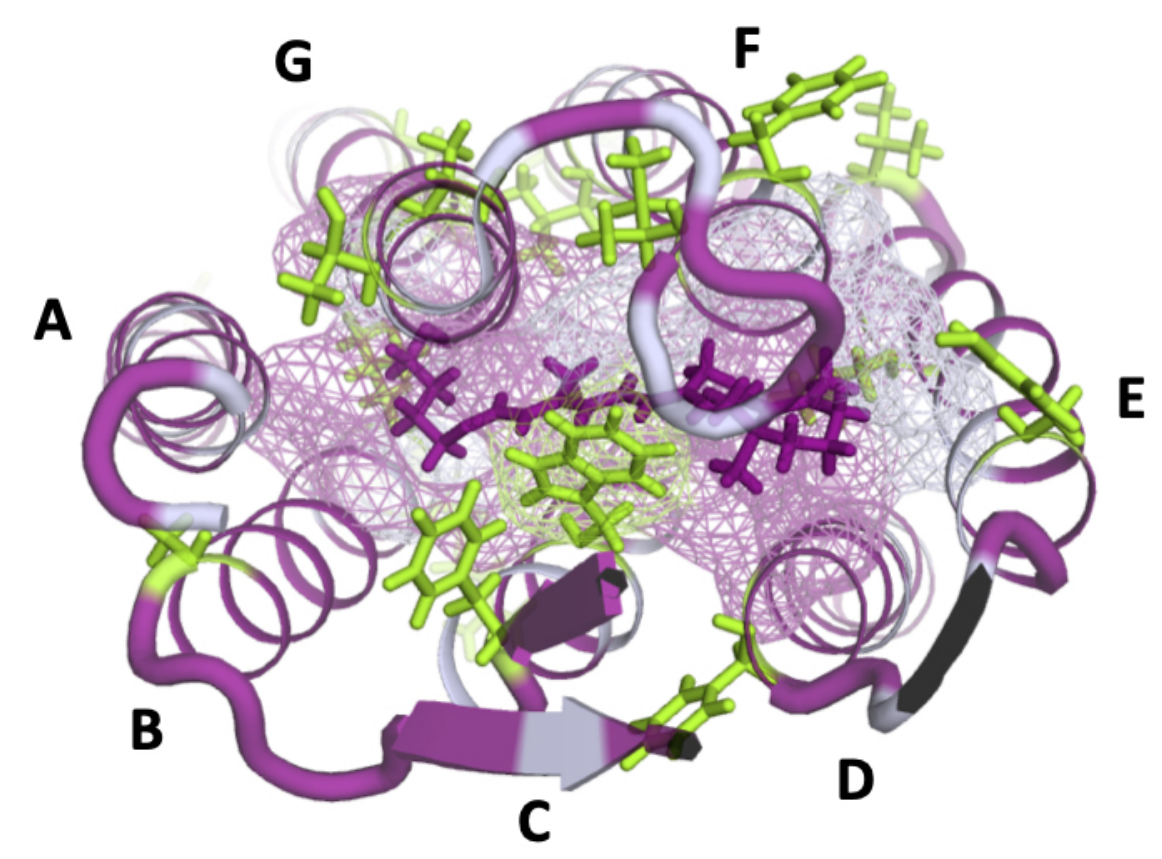

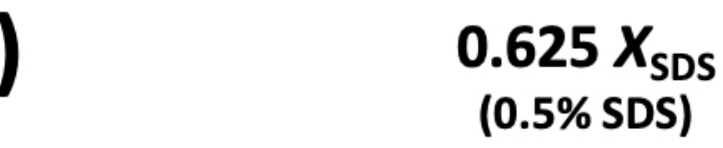

(0.5\% SDS)
$0.834 X_{\text {SDS }}$

(1.5\% SDS)
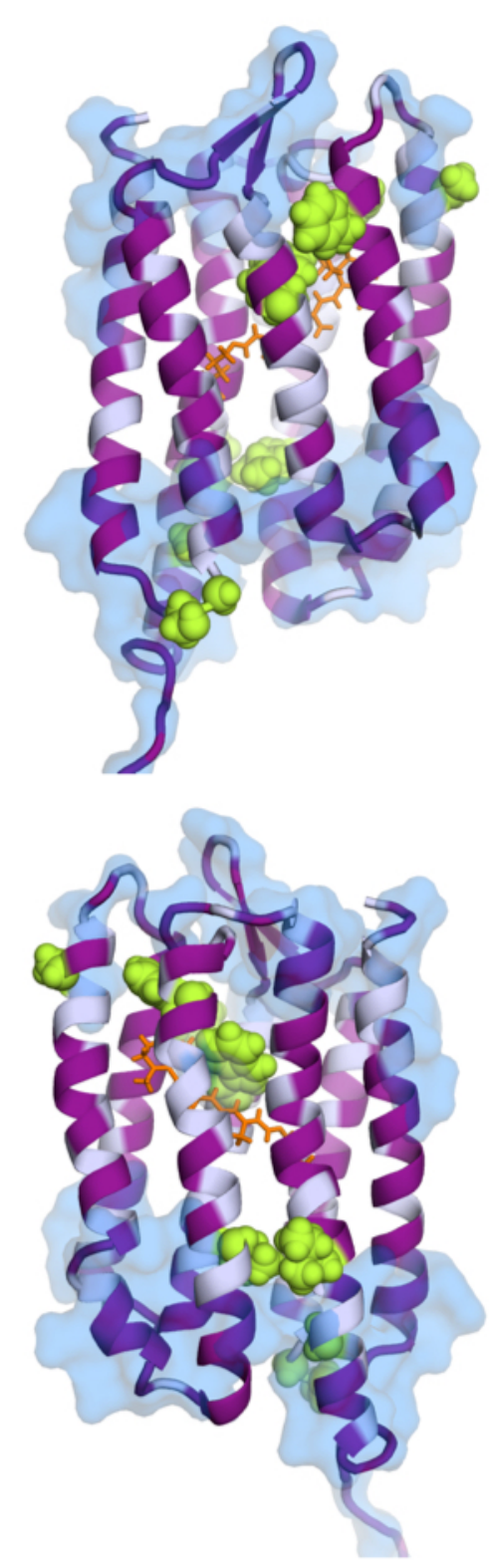

$0.870 X_{\text {SDS }}$

(2.0\% SDS)
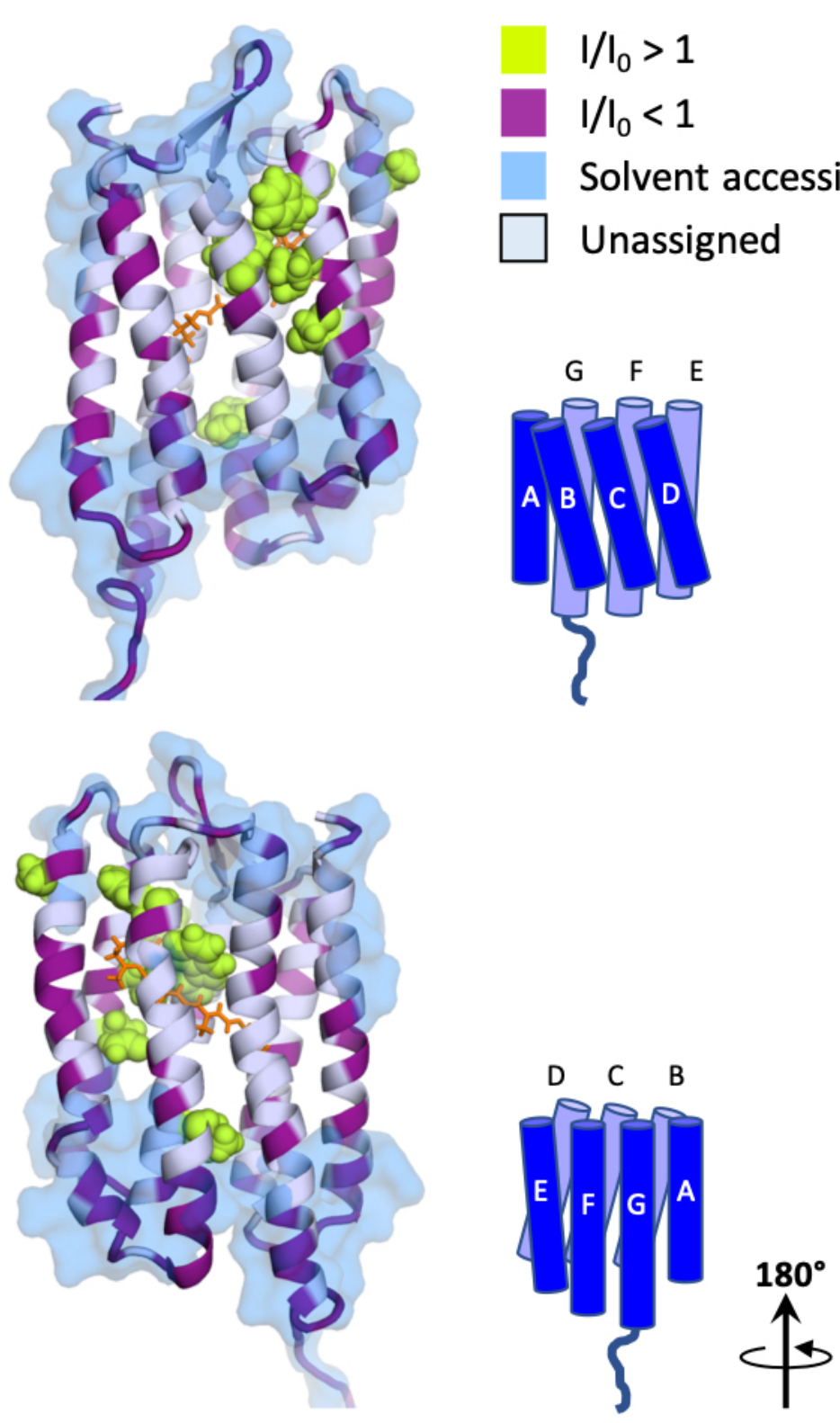

$\mathrm{I} / \mathrm{I}_{0}>1$
$\mathrm{I} / \mathrm{I}_{0}<1$

Solvent accessible in $0 X_{\text {SDS }}$

$\square$ Unassigned

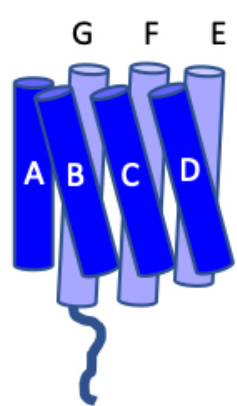

$180^{\circ}$

个

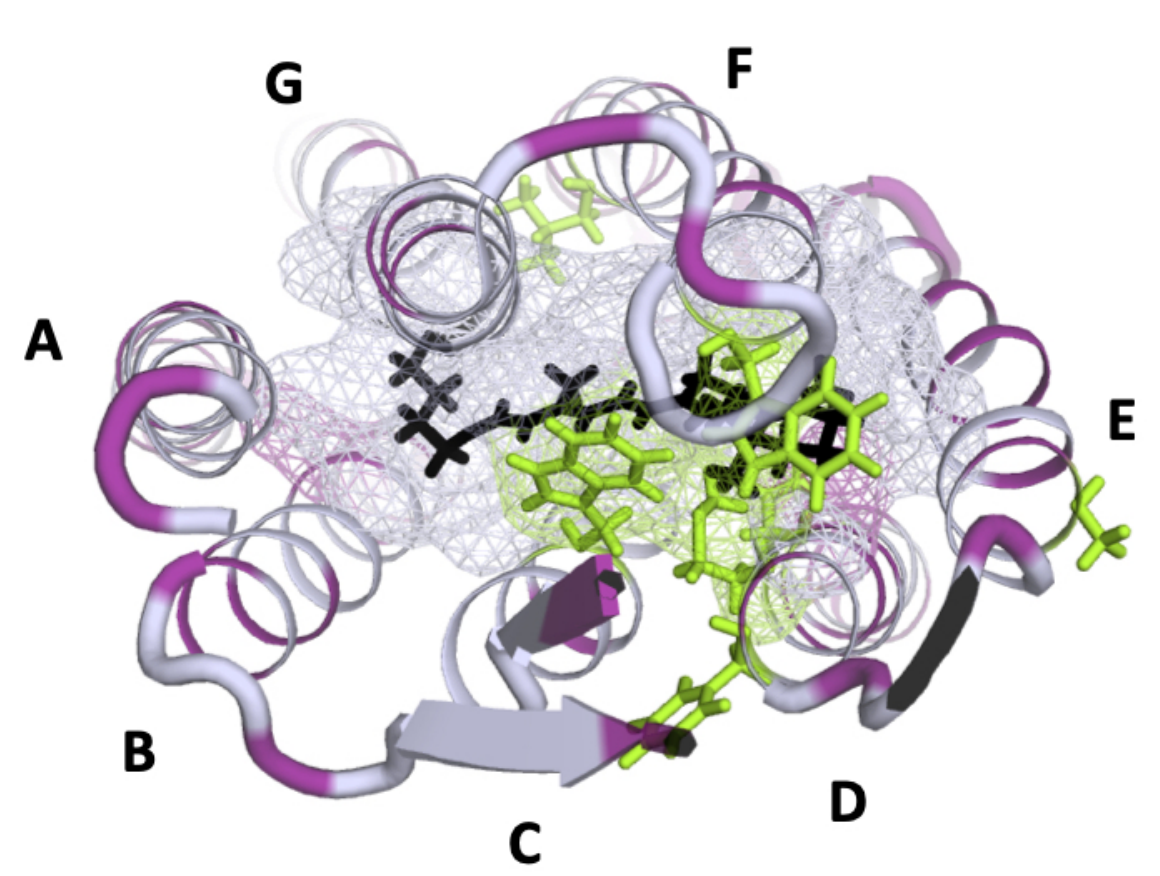

(2.0\% SDS) 

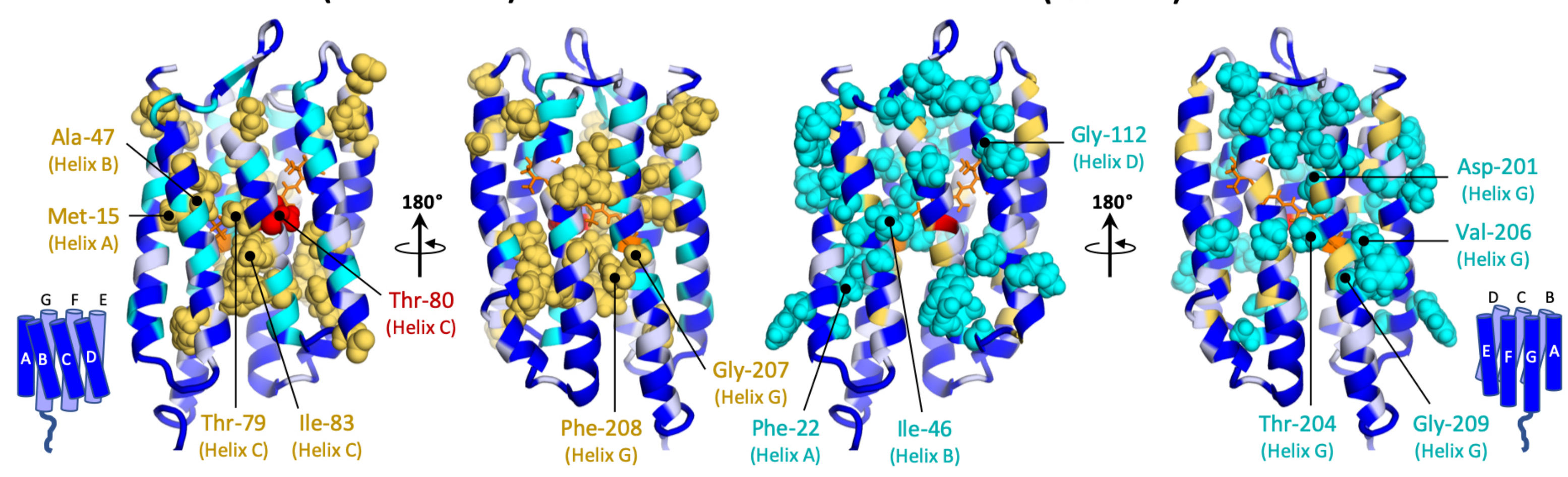

(b)

$$
\begin{gathered}
0.769-0.834 X_{\text {SDS }} \\
(1.0-1.5 \% \text { SDS })
\end{gathered}
$$

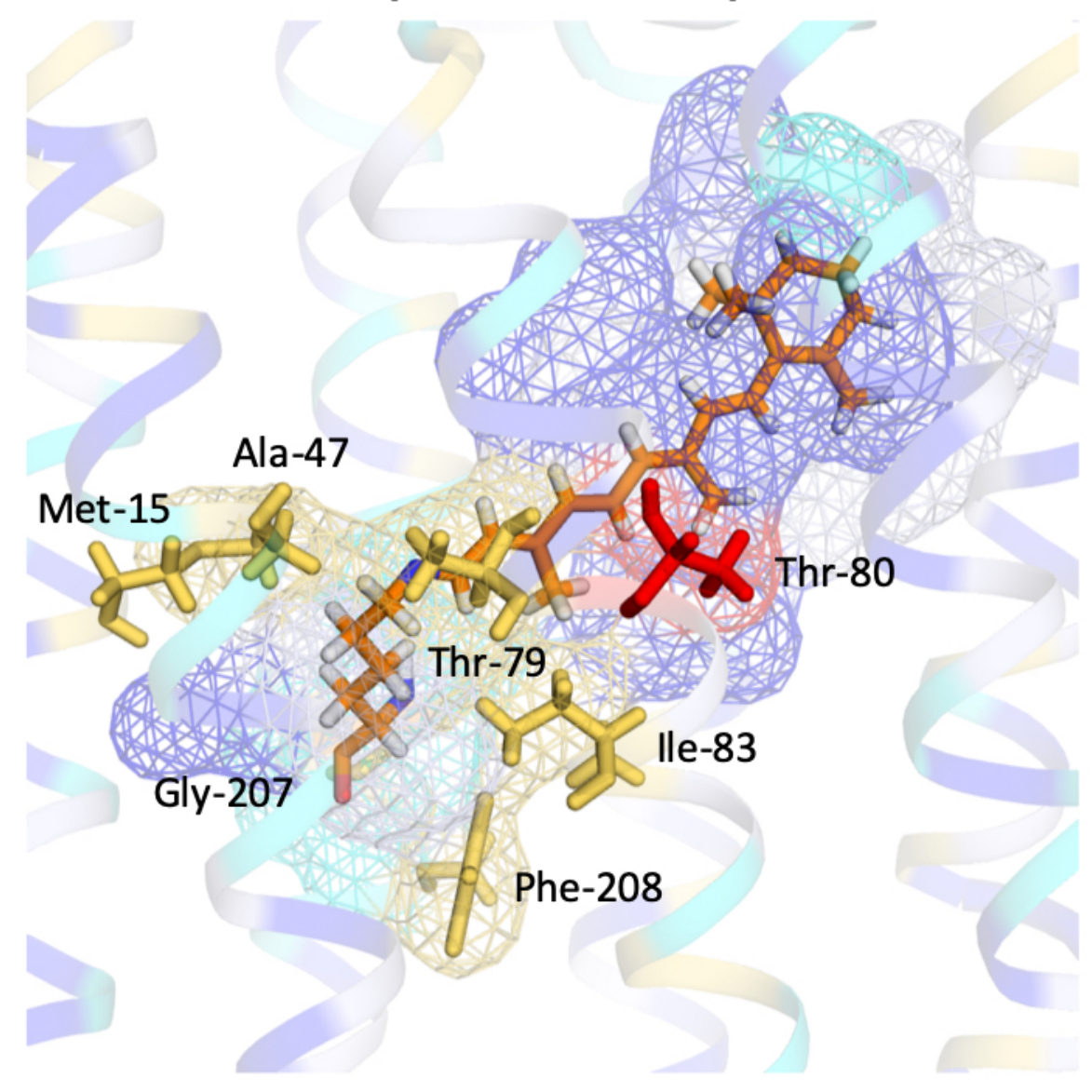

\section{$0.870 X_{\text {SDS }}$ \\ (2.0\% SDS)}

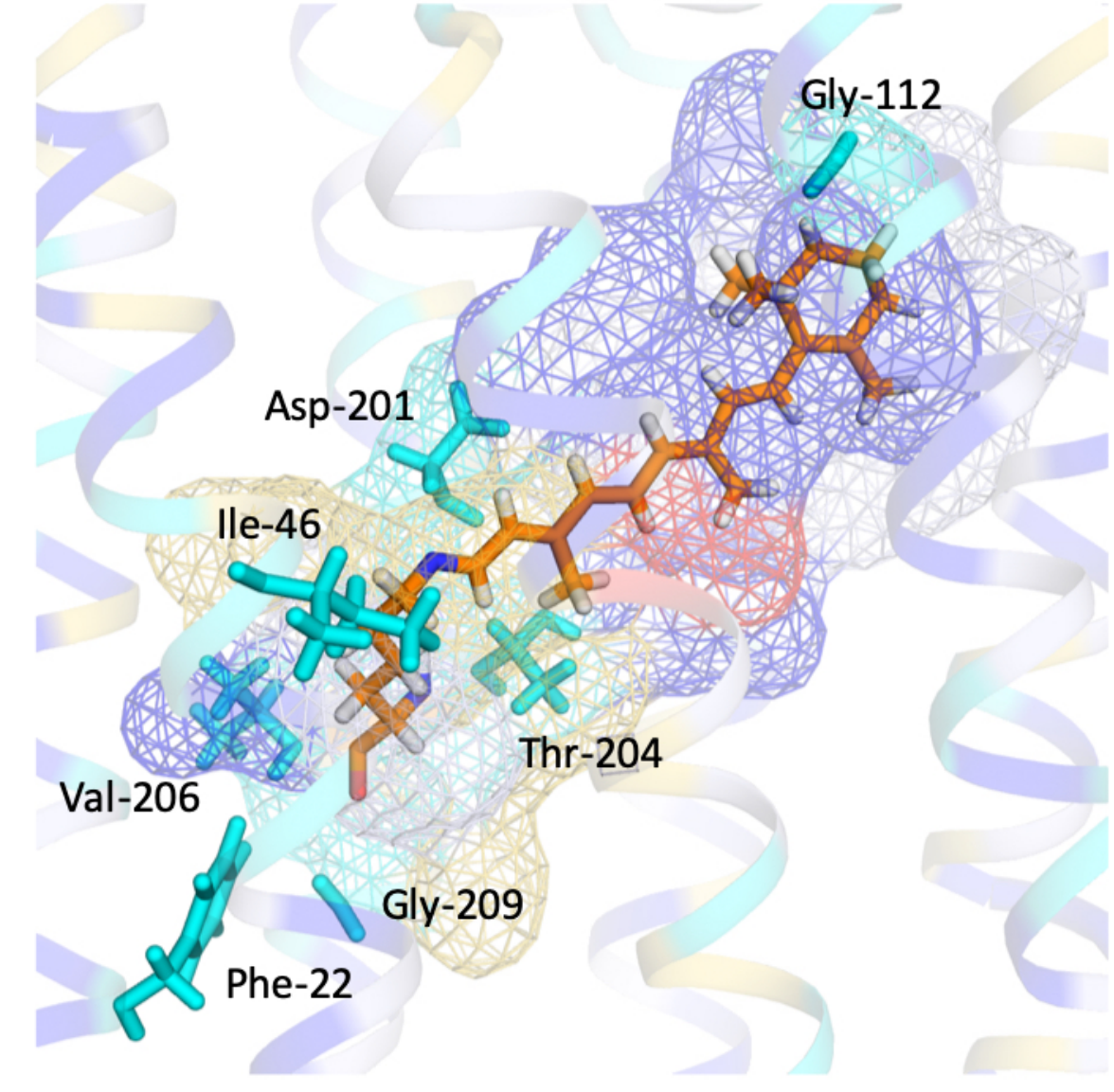

(c)

Extracellular view

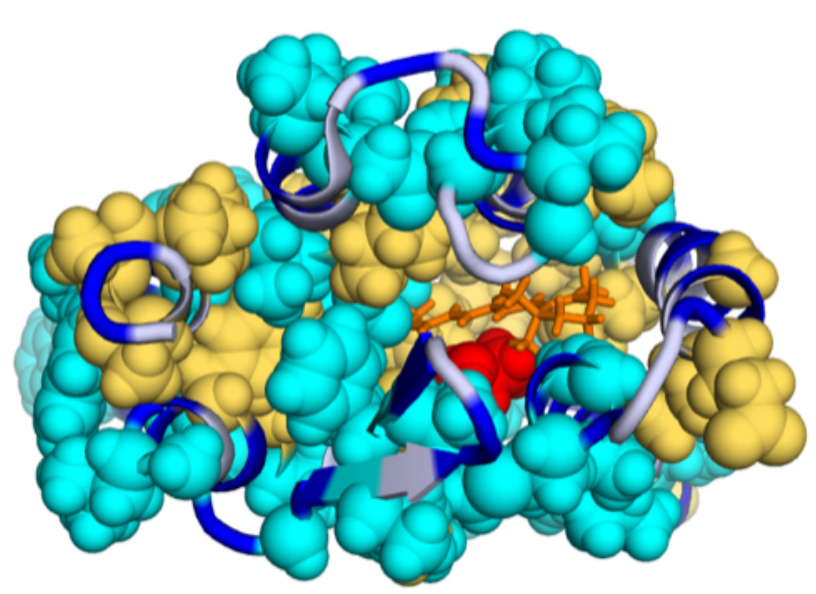

Cytoplasmic view

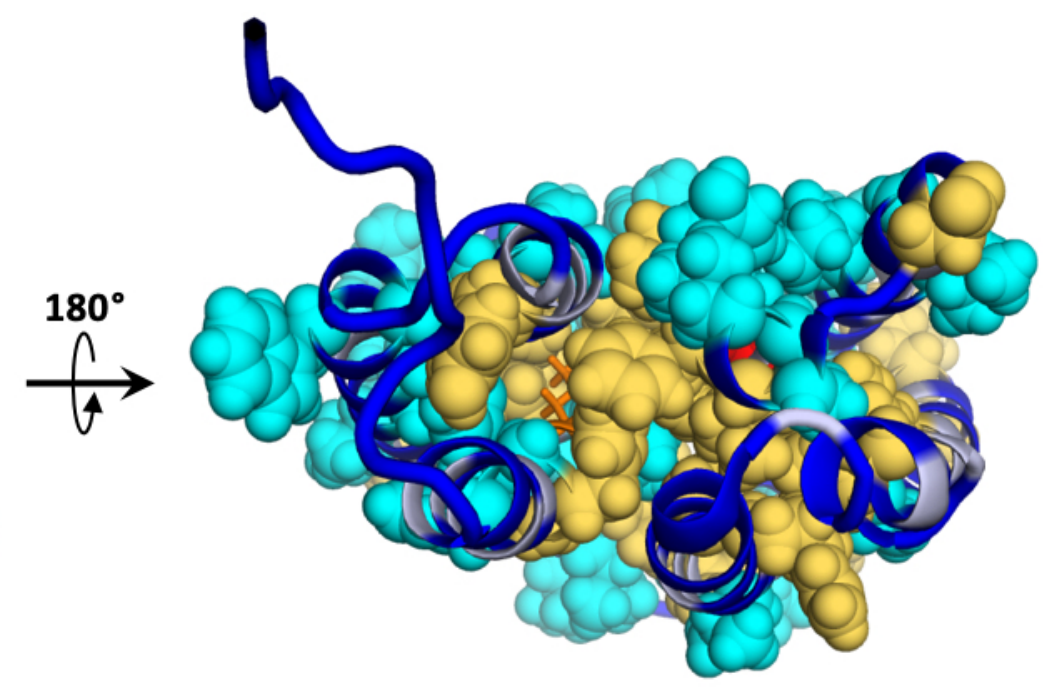

\section{Unobservable at:}

$0.769 X_{\text {SDS }}(1.0 \%$ SDS $)$

$\square 0.834 X_{\text {SDS }}(1.5 \%$ SDS $)$

$0.870 X_{\text {SDS }}(2.0 \%$ SDS $)$

Observable at $\geq 0.893 X_{\text {SDS }}(2.5 \%$ SDS $)$

Unassigned 
(a)

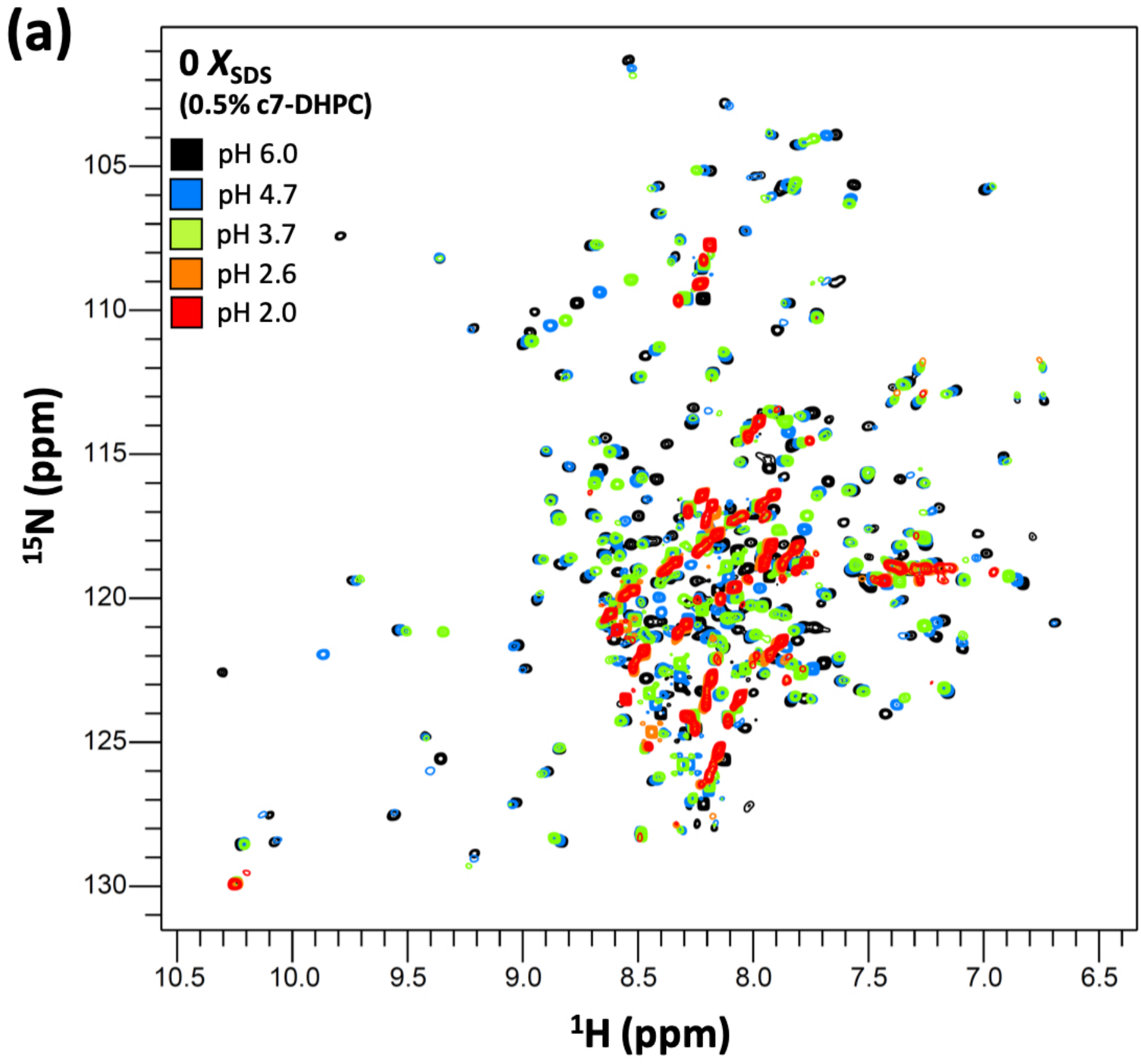

(b)

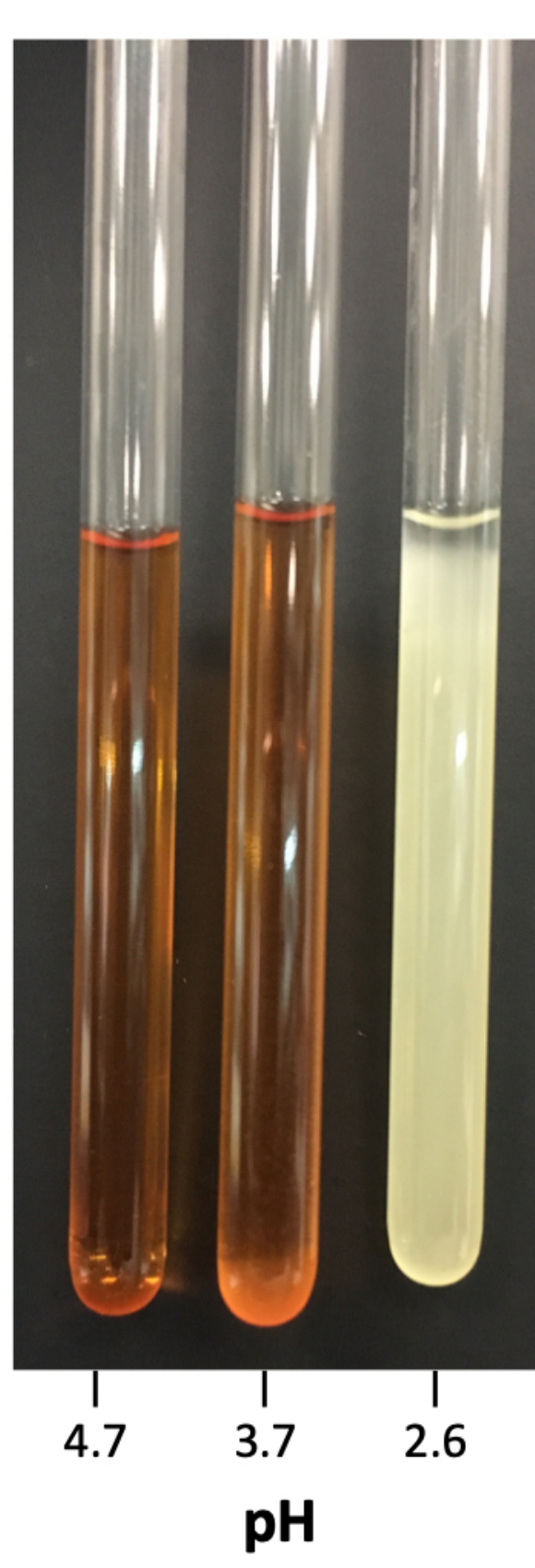

(d) Trp-178 (Helix F)

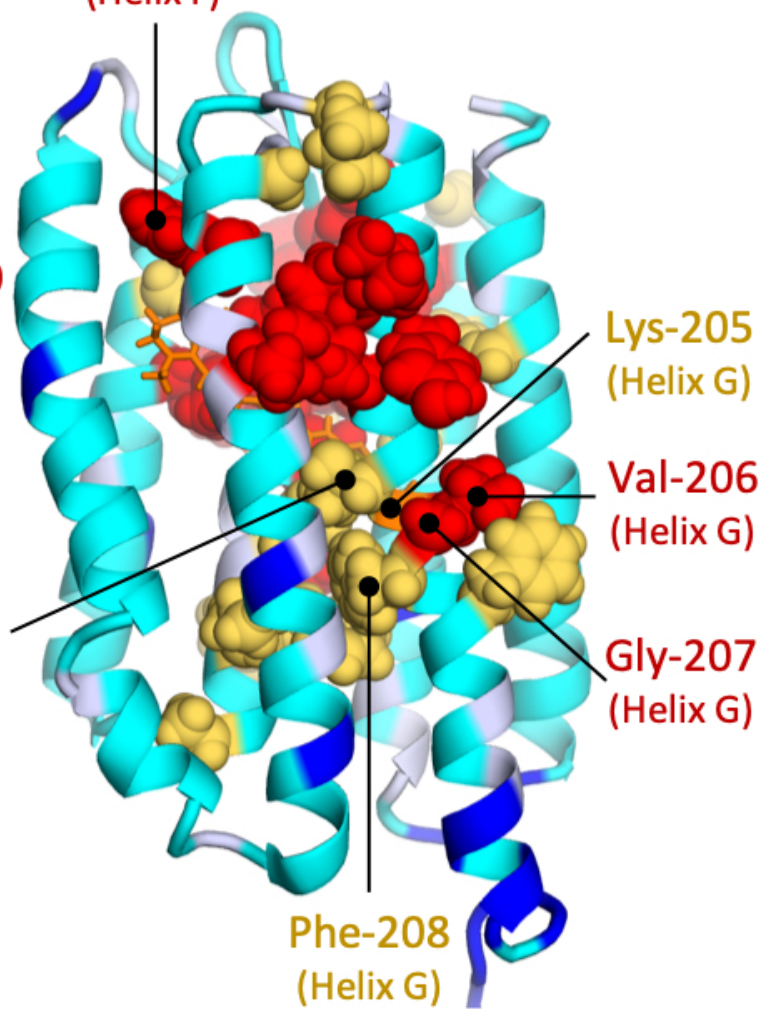

Unobservable at:

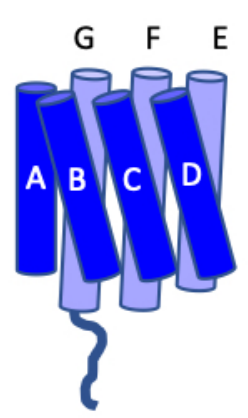

$\leq \mathrm{pH} 4.7$

$\leq \mathrm{pH} 3.7$

$\leq \mathrm{pH} 2.6$

Observable at $\mathrm{pH} 2.0$ Unassigned

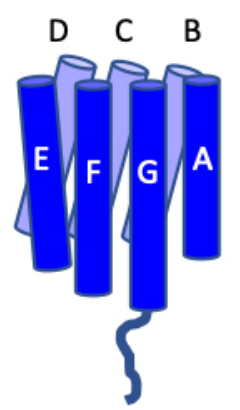

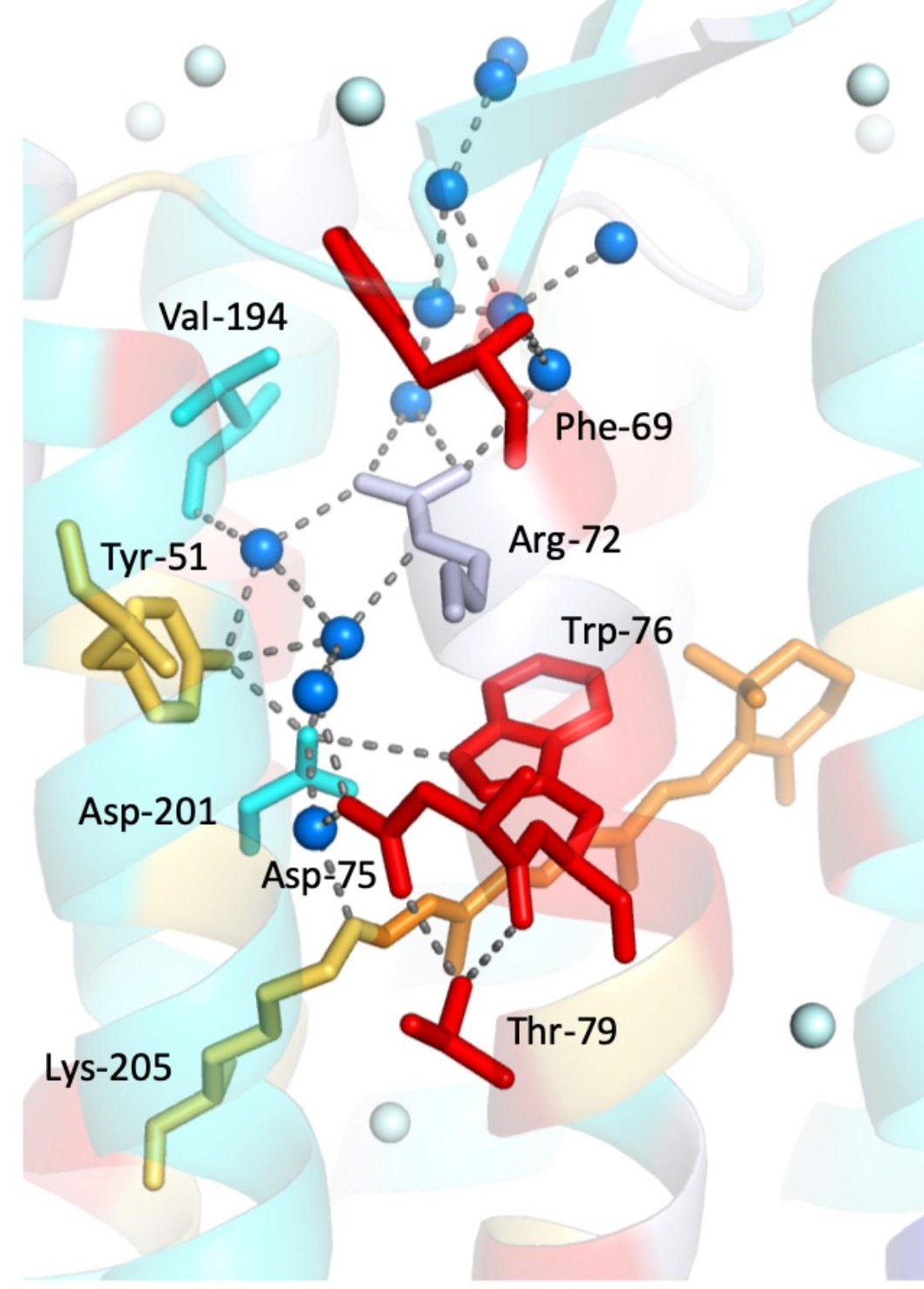




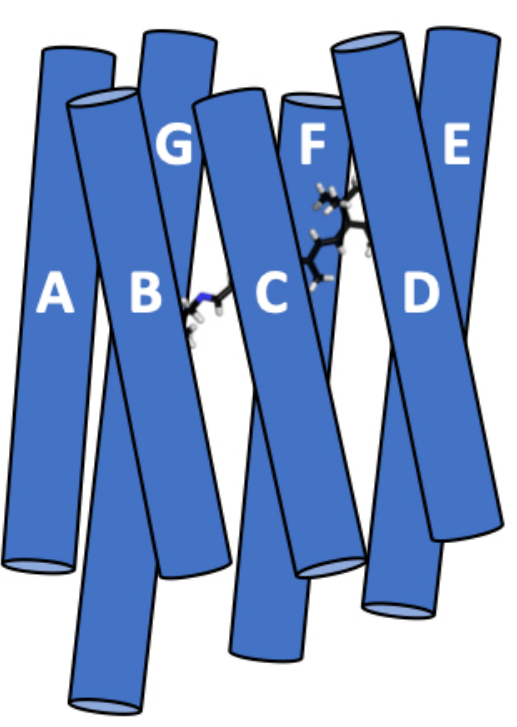

Folded pSRII in c7-DHPC, pH 6.0
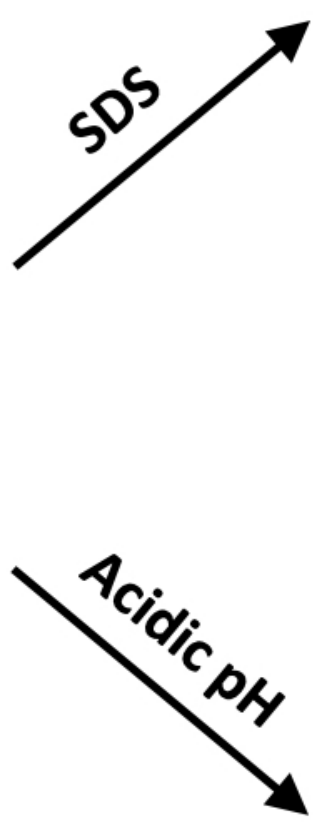
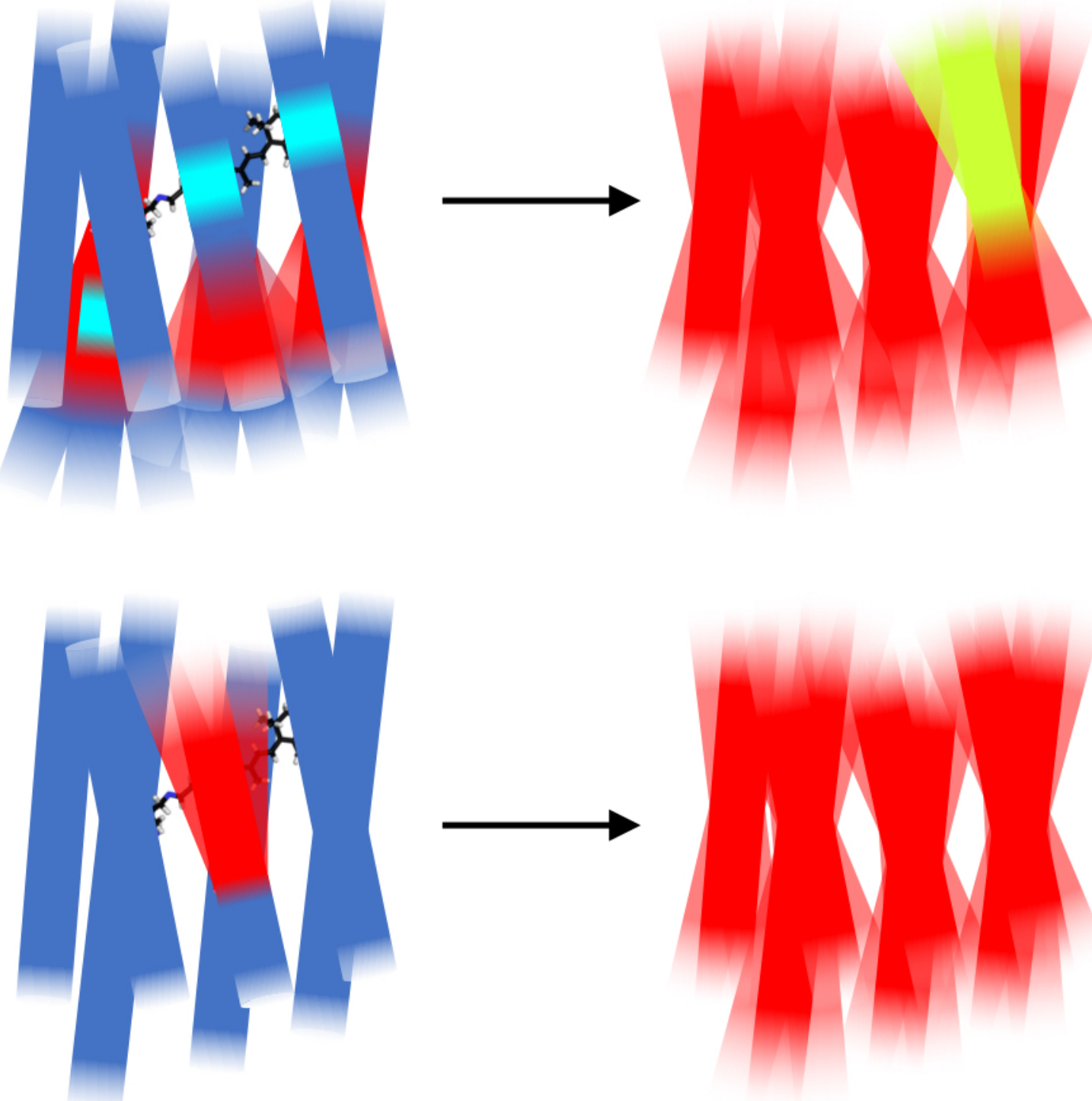

Structural change within retinal-binding pocket

$\mu s$-to-ms timescale conformational exchange

Fast local dynamics 


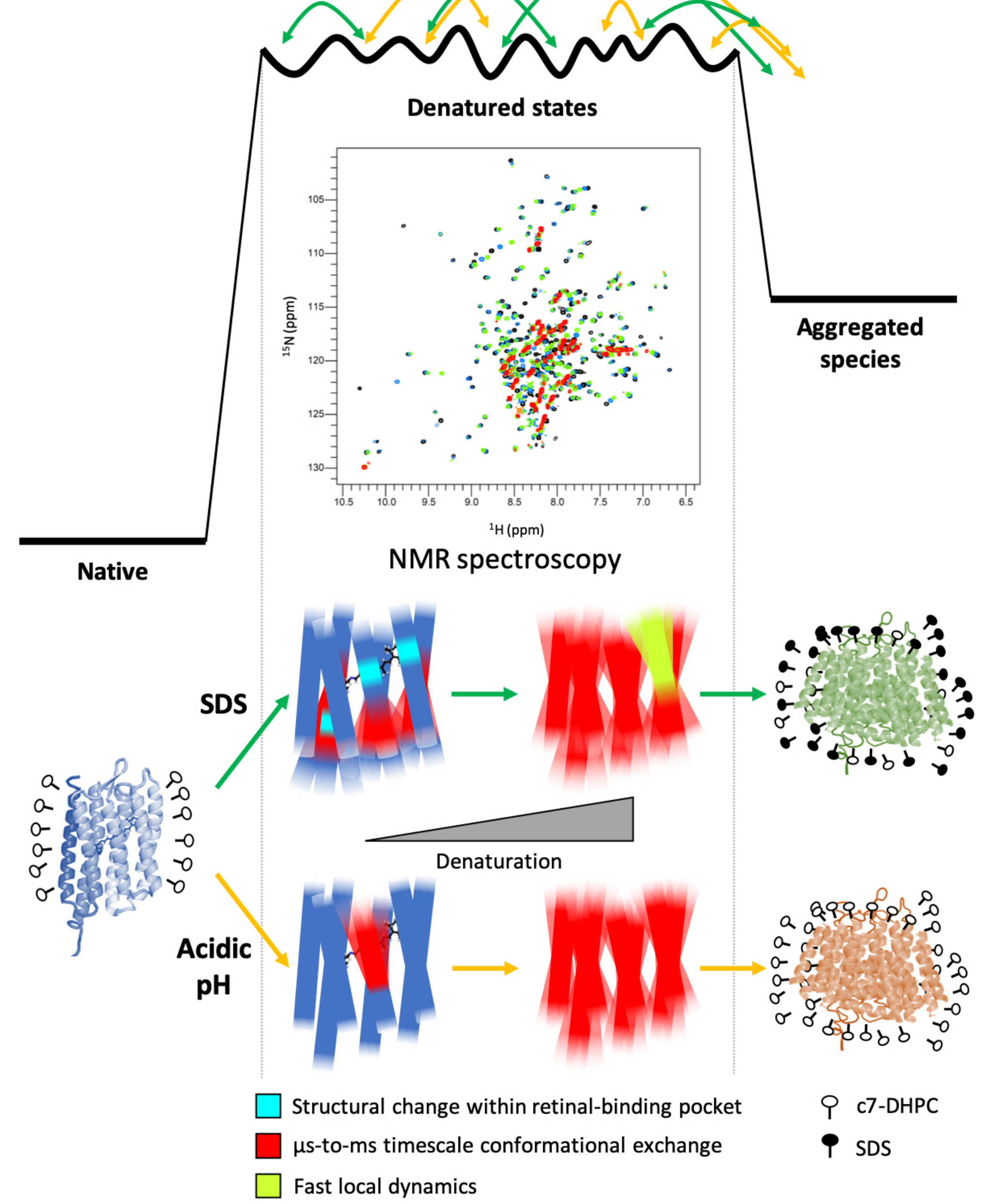




\section{Supplementary Information}

\section{Characterisation of denatured states of sensory rhodopsin II by solution-state NMR}

Yi Lei $\operatorname{Tan}^{1}$, James Mitchell ${ }^{2,3}$, Judith Klein-Seetharaman ${ }^{2,4}$, Daniel Nietlispach ${ }^{1 *}$

${ }^{1}$ Department of Biochemistry, 80 Tennis Court Road, University of Cambridge, CB2 1GA, United Kingdom

${ }^{2}$ Biomedical Sciences Division, Warwick Medical School, University of Warwick, Coventry, CV4 7AL, United Kingdom

${ }^{3}$ Present address: School of Life Sciences, University of Nottingham, NG7 2UH, United Kingdom

${ }^{4}$ Present address: Department of Chemistry, Integrative Graduate Program in Quantitative Biosciences and Engineering, Colorado School of Mines, 1500 Illinois Street, Golden CO 80401

*To whom correspondence should be addressed: Daniel Nietlispach, Department of Biochemistry, 80 Tennis Court Road, University of Cambridge, CB2 1GA, United Kingdom. Phone: +44 1223 766023, Fax: +44 1223 766002, Email: dn206@cam.ac.uk 


\section{Supplementary information}

\section{Spectral quality}

Our previous study [1] shows that SDS-denatured pSRII is susceptible to aggregation if left exposed to SDS for durations of hours to days. To understand how spectral quality is affected by pSRII denaturation and aggregation at $35{ }^{\circ} \mathrm{C}$, time-dependent changes were studied in 2D $\left[{ }^{1} \mathrm{H},{ }^{15} \mathrm{~N}\right]$ BEST-TROSY spectra of pSRII in $0.834 \mathrm{X}_{\mathrm{SDS}}(1.5 \%$ SDS in $0.5 \% \mathrm{c} 7-\mathrm{DHPC})$ (Figure S2a) and in ${ }^{1} \mathrm{H}$ NMR spectra of pSRII in $0.971 X_{\text {SDS }}$ (10\% SDS in $0.5 \%$ c7-DHPC) (Figure S2b). Both samples showed complete retinal Schiff base hydrolysis and became pale yellow in colour (absorbance $\lambda_{\max }=390 \mathrm{~nm}$ ) after $21 \mathrm{~h}$. Comparison of the BEST-TROSY and ${ }^{1} \mathrm{H}$ spectra over time showed very small decrease in peak intensities within $6 \mathrm{~h}$ of initial exposure to SDS, while a more pronounced decrease in peak intensities was observed from $6 \mathrm{~h}$ onwards, indicative of aggregation. This is further supported by the decrease in $\alpha$-helical content determined from changes in MRE at $222 \mathrm{~nm}$ measured by circular dichroism and by the appearance of oligomeric bands observed by SDS-PAGE from $6 \mathrm{~h}$ onwards at $35^{\circ} \mathrm{C}$ (Figures S2c-d). Hence, NMR spectra were monitored for a maximum of $6 \mathrm{~h}$ to minimise the effects of aggregation on spectral quality. Changes in MRE at $222 \mathrm{~nm}$ measured by circular dichroism also showed that unfolding occurred to the same extent at both $25^{\circ} \mathrm{C}$ and $35{ }^{\circ} \mathrm{C}$ (Figure S2c), thus indicating that the studies reported in this paper are comparable against our previous biophysical measurements [1].

Although chemical shift changes can be plotted against ligand concentration in proteinobserved NMR ligand titration studies and parameters including binding affinity can be extracted from saturation ligand-binding curves [2,3], this approach proved to be challenging for studying SDS denaturation and acid denaturation of pSRII because of various reasons. Firstly, it is expected that SDS would integrate into c7-DHPC micelles to form mixed micelles rather than engage in specific ligand-binding interactions with backbone amides. Secondly, protein structural changes due to denaturation would lead to deviations from the smooth hyperbolic saturation ligand-binding curve as the denatured states interact with the surrounding detergent micelle in a different manner compared to the folded state. Finally, the absence of data points at high $X_{S D S}$ and low $\mathrm{pHs}$ due to resonances becoming unobservable with excessive line broadening prevents the observation of the plateau of the binding curve for many residues, and hence attempts at fitting such curves would be inaccurate.

The prominence of intermediate timescale exchange in SDS-denatured and acid-denatured pSRII has limited the extent of quantitative data interpretation to a rather simplified and qualitative treatment of the NMR titration data in this paper. It has also precluded the utilisation of more quantitative methods such as 2D NMR lineshape analysis [4] and principal component analysis [5,6] for analysing chemical shift changes of pSRII, because of the reliance of such methods on identifying the end-point of the titration. Altogether, the limitations in NMR data quality and results interpretation emphasise the challenges underlying high resolution structural studies on membrane protein folding. 


\section{Changes in chemical shifts and peak intensities for tryptophan side-chains of pSRII in SDS}

Side-chains are exposed to different chemical environments compared to backbone amides (Figure S6a), and are directly involved in inter-helical interactions, $\mathrm{H}$-bond networks and protein-retinal packing. Monitoring changes in tryptophan side-chain indoles by NMR therefore provides extra probes for tertiary structure changes.

Like the backbone amides, pSRII tryptophan side-chain indole resonances showed complex chemical shift changes during denaturation mediated by different $X_{\text {SDS }}$ (Figure S6b). Non-linear changes in resonance positions were observed for all tryptophan side-chain indole resonances, suggesting fast exchange between different side-chain conformations. Alternative peaks were observed for Trp-9 and Trp-24, both in helix A, starting from 0.870 $X_{\text {SDS }}(2 \%$ SDS in $0.5 \%$ c7-DHPC), indicating that conformational exchange between different side-chain conformations transitioned from the fast exchange regime to the slow exchange regime beyond the unfolding transition.

Side-chain $\Delta \delta\left({ }^{1} \mathrm{H},{ }^{15} \mathrm{~N}\right)$ correlated with the locations of the tryptophan residues (Figure S6c). Trp-76 and Trp-171, which pack directly against the retinal, showed the smallest $\Delta \delta\left({ }^{1} \mathrm{H},{ }^{15} \mathrm{~N}\right)$ that correlated linearly against $X_{\text {SDS }}$ only up to $0.728 X_{\text {SDS, }}$ indicating early onset of structural changes within the retinal-binding pocket starting from $>0.728 X_{\text {SDS. }}$ Trp-60 and Trp-178 both showed bigger $\Delta \delta\left({ }^{1} \mathrm{H},{ }^{15} \mathrm{~N}\right)$ that correlated linearly against $X_{\mathrm{SDS}}$. This likely reflects their greater accessibility to SDS as Trp-60 is located in the B/C loop while Trp-178 is located near the extracellular end of helix F. Trp-9 and Trp-24, both of which face the detergent micelle, experienced the largest $\Delta \delta\left({ }^{1} \mathrm{H},{ }^{15} \mathrm{~N}\right) . \Delta \delta\left({ }^{1} \mathrm{H},{ }^{15} \mathrm{~N}\right)$ of Trp-24 correlated linearly against $X_{\mathrm{SDS}}$ up to $0.769 X_{\mathrm{SDS}}(1 \% \mathrm{SDS}$ in $0.5 \% \mathrm{C7}-\mathrm{DHPC}$ ) but correlated linearly against the absolute concentration of SDS in $0.834-0.909 X_{\text {SDS }}(1.5-3.0 \%$ SDS in $0.5 \%$ c7-DHPC) (Figure S6d). This suggests that the side-chain of Trp-24 interacts with micellar SDS in low $X_{\text {SDS }}$ but interacts with non-micellar SDS above the unfolding transition. Compared to those of other tryptophan side-chains, $\Delta \delta\left({ }^{1} \mathrm{H},{ }^{15} \mathrm{~N}\right)$ of Trp-9 did not correlate very well against $X_{\mathrm{SDS}}$ or the absolute concentration of SDS, possibly suggesting that Trp-9 interacted with both micellar and non-micellar SDS.

Consistent decreases in peak intensities were observed with increasing $X_{\text {SDS }}$ for most tryptophan side-chain indoles (Figure S6e), indicating that side-chain motions are becoming increasingly dominated by $\mu$ s-to-ms timescale motions. Only Trp-24 side-chain indole

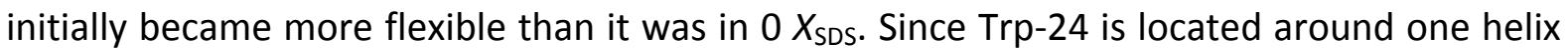
turn away from the cytoplasmic end of helix $A$, this observation could reflect slight unravelling at the end of helix $A$. Beyond $0.834 X_{\text {SDS }}$, the alternative conformations of Trp- 9 and Trp-24 side-chain indoles showed increased fast exchange with further increases in $X_{\text {SDS }}$ (Figures S6b \& S6e). This highlights the heterogeneous nature of the denatured states ensemble.

Summarising the above, tryptophan side-chains generally experienced little changes in their chemical environment, consistent with the protein remaining embedded in a micellar environment. Only side-chains which face the detergent micelle experienced greater changes in chemical environment due to incorporation of SDS into the micelles. Side-chain motions became increasingly dominated by $\mu$ s-to-ms timescale motions in increasing $X_{\text {SDS. }}$. 


\section{Supplementary references}

[1] Y.L. Tan, J. Mitchell, J. Klein-Seetharaman, D. Nietlispach, Characterization of Denatured States and Reversible Unfolding of Sensory Rhodopsin II, J. Mol. Biol. 430 (2018) 4068-4086. doi:10.1016/j.jmb.2018.07.031.

[2] R.E. London, Chemical-shift and linewidth characteristics of reversibly bound ligands, J. Magn. Reson. Ser. A. 104 (1993) 190-196. doi:10.1006/jmra.1993.1208.

[3] M.P. Williamson, Using chemical shift perturbation to characterise ligand binding, Prog. Nucl. Magn. Reson. Spectrosc. 73 (2013) 1-16. doi:10.1016/j.pnmrs.2013.02.001.

[4] C.A. Waudby, A. Ramos, L.D. Cabrita, J. Christodoulou, Two-dimensional NMR lineshape analysis, Sci. Rep. 6 (2016) 24826. doi:10.1038/srep24826.

[5] K. Sakurai, Y. Goto, Principal component analysis of the pH-dependent conformational transitions of bovine beta-lactoglobulin monitored by heteronuclear NMR, Proc. Natl. Acad. Sci. 104 (2007) 15346-15351. doi:10.1073/pnas.0702112104.

[6] T. Konuma, Y.H. Lee, Y. Goto, K. Sakurai, Principal component analysis of chemical shift perturbation data of a multiple-ligand-binding system for elucidation of respective binding mechanism, Proteins Struct. Funct. Bioinforma. 81 (2013) 107-118. doi:10.1002/prot.24166. 


\section{Supplementary figures}

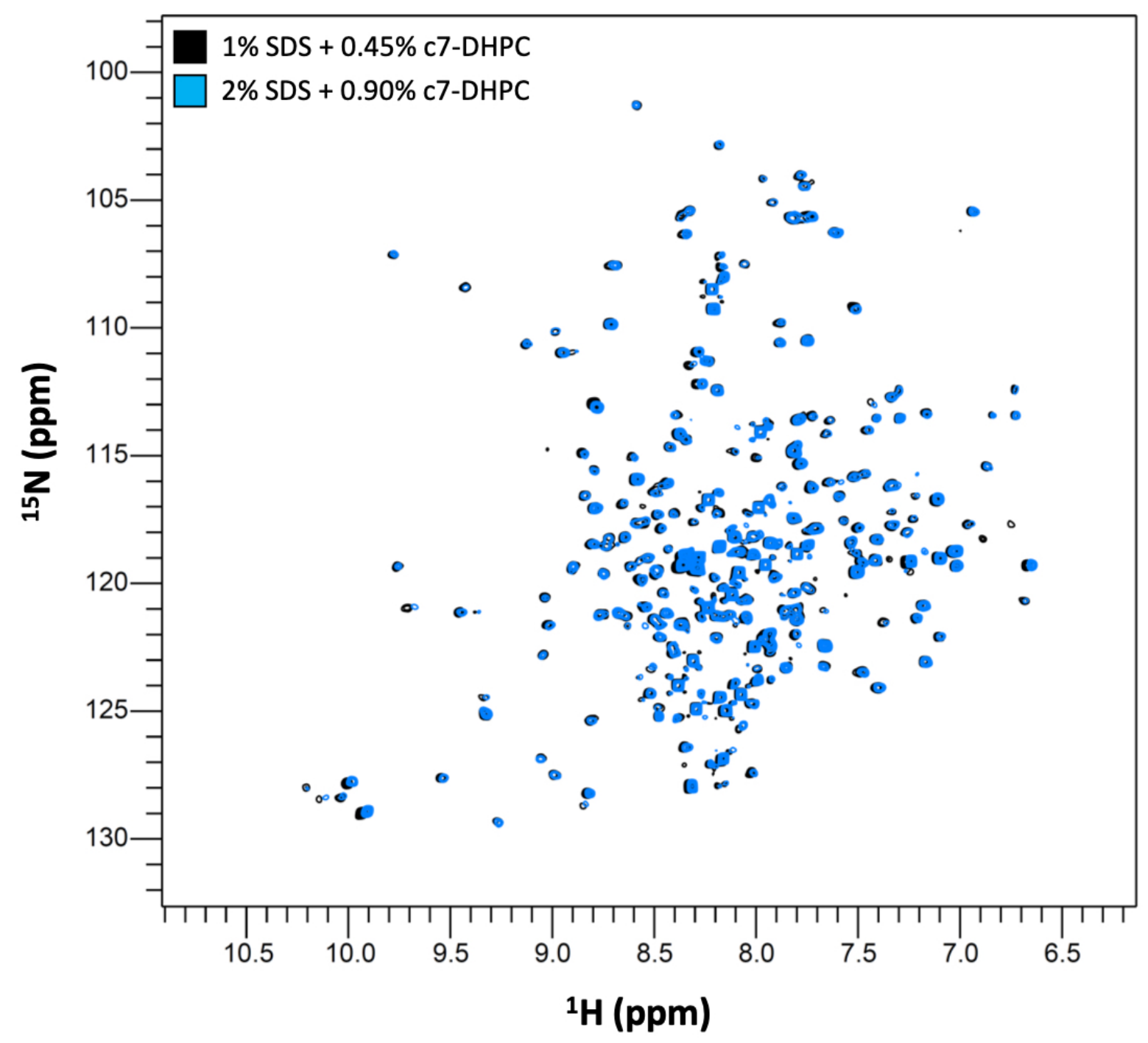

Figure S1: 2D $\left[{ }^{1} \mathrm{H},{ }^{15} \mathrm{~N}\right]$ BEST-TROSY spectra recorded in $0.769 X_{\mathrm{SDS}}$ with different molar concentrations of SDS and C7-DHPC

Spectra were recorded with $94 \mu \mathrm{M}{ }^{15} \mathrm{~N}$-pSRII on an $800 \mathrm{MHz}$ spectrometer at $308 \mathrm{~K}$, with 240 scans. All samples contained TSP as internal chemical shift reference. 
(a)

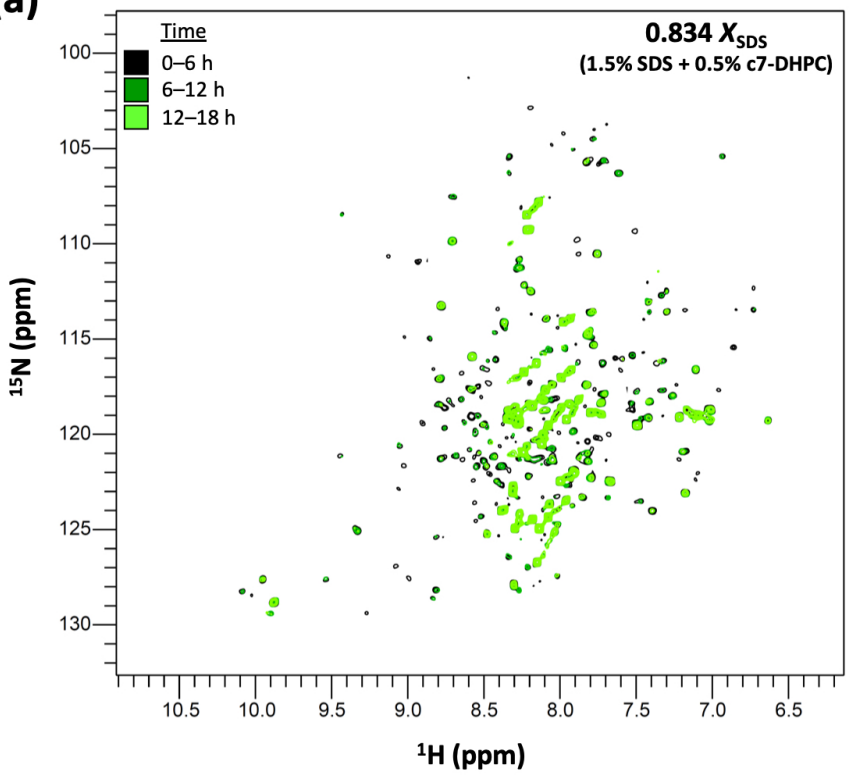

(c)

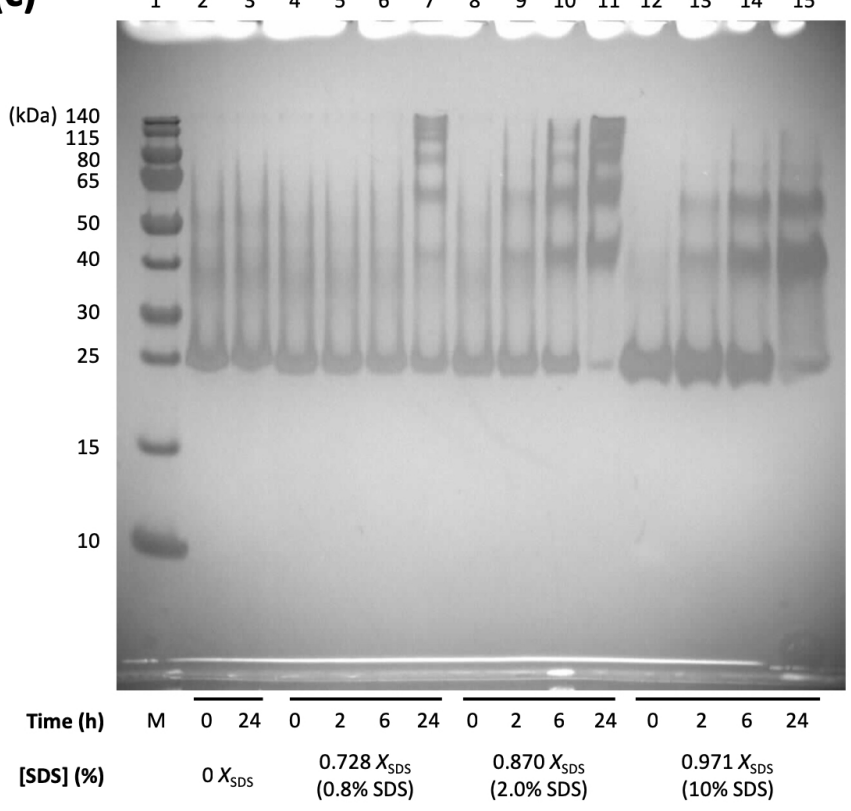

(b)
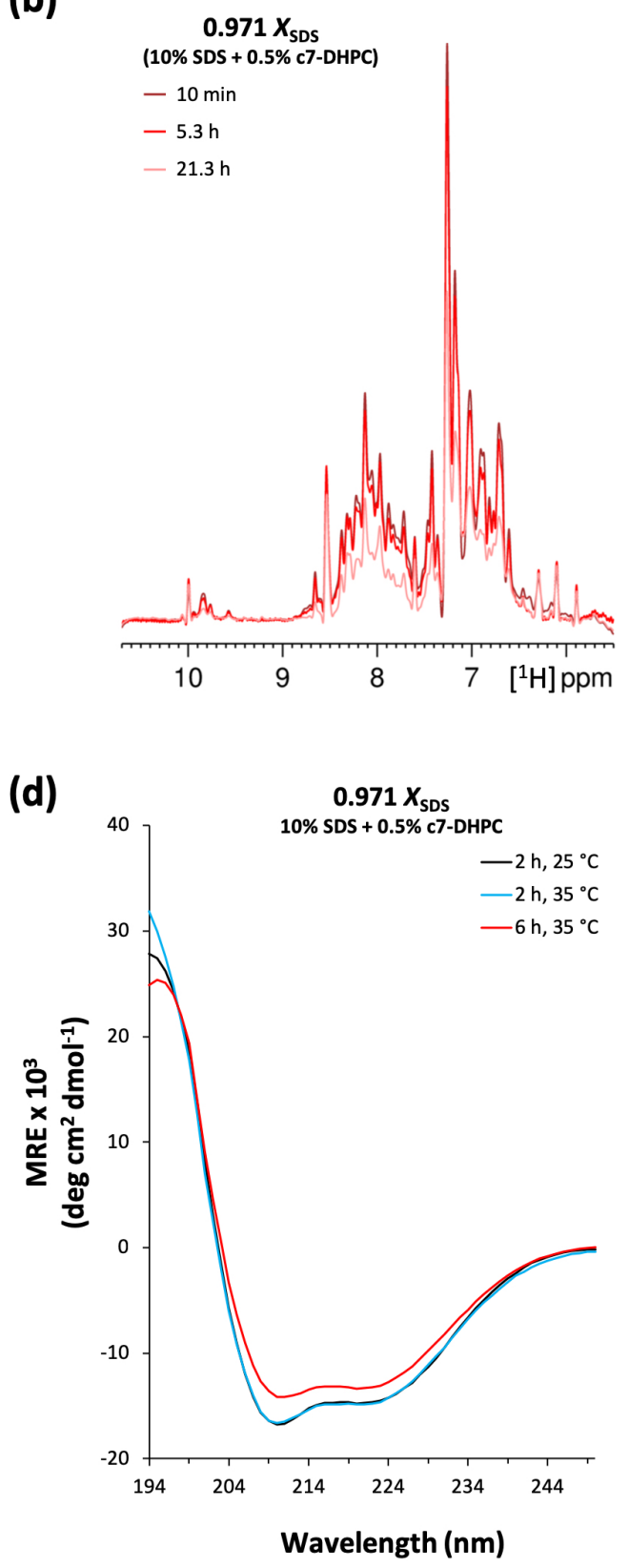

Figure S2: (a) 2D $\left[{ }^{1} \mathrm{H},{ }^{15} \mathrm{~N}\right]$ BEST-TROSY spectra recorded for $83 \mu \mathrm{M}{ }^{15} \mathrm{~N}$-pSRII in $0.834 \mathrm{X}_{\mathrm{SDS}}$ (1.5\% SDS in 0.5\% c7-DHPC) for increasing amount of time. All spectra were recorded on an $800 \mathrm{MHz}$ spectrometer at $308 \mathrm{~K}$, with 240 scans. (b) ${ }^{1} \mathrm{H}$ NMR of backbone amides and sidechain $\mathrm{NH}$ groups for $83 \mu \mathrm{M}$ pSRII in $0.971 X_{\text {SDS }}(10 \%$ SDS in 0.5\% c7-DHPC) for $10 \mathrm{~min}, 5.3 \mathrm{~h}$ or $21.3 \mathrm{~h}$. All spectra were recorded at $308 \mathrm{~K}$ with 512 scans, and processed by apodization of the FID using a squared sine window function shifted by $90^{\circ}$ followed by an exponential line broadening of $1 \mathrm{~Hz}$. All NMR samples contained TSP as internal chemical shift reference. (c) Silver-stained $12 \%$ SDS-PAGE showing increased aggregation of pSRII with increasing $X_{\text {SDS }}$ over prolonged unfolding times ( $0 \mathrm{~min}, 2 \mathrm{~h}, 6 \mathrm{~h}, 24 \mathrm{~h}$ ). Lane 1, molecular weight marker; lanes 2-3, $0 X_{\text {SDS }}\left(0.5 \%\right.$ c7-DHPC); lanes 4-7, $0.728 X_{\text {SDS }}(0.8 \%$ SDS + 0.5\% c7-DHPC); lanes 8$11,0.870 X_{\text {SDS }}\left(2.0 \%\right.$ SDS + 0.5\% c7-DHPC); lanes $12-15,0.971 X_{\text {SDS }}(10 \%$ SDS + 0.5\% c7DHPC). (d) Circular dichroism (MRE at $222 \mathrm{~nm}$ ) for $7 \mu \mathrm{M}(0.2 \mathrm{mg} / \mathrm{ml})$ pSRII in $0.971 X_{\text {SDS }}(10 \%$ SDS + 0.5\% c7-DHPC) at $25^{\circ} \mathrm{C}$ or $35^{\circ} \mathrm{C}$ for up to $6 \mathrm{~h}$. 


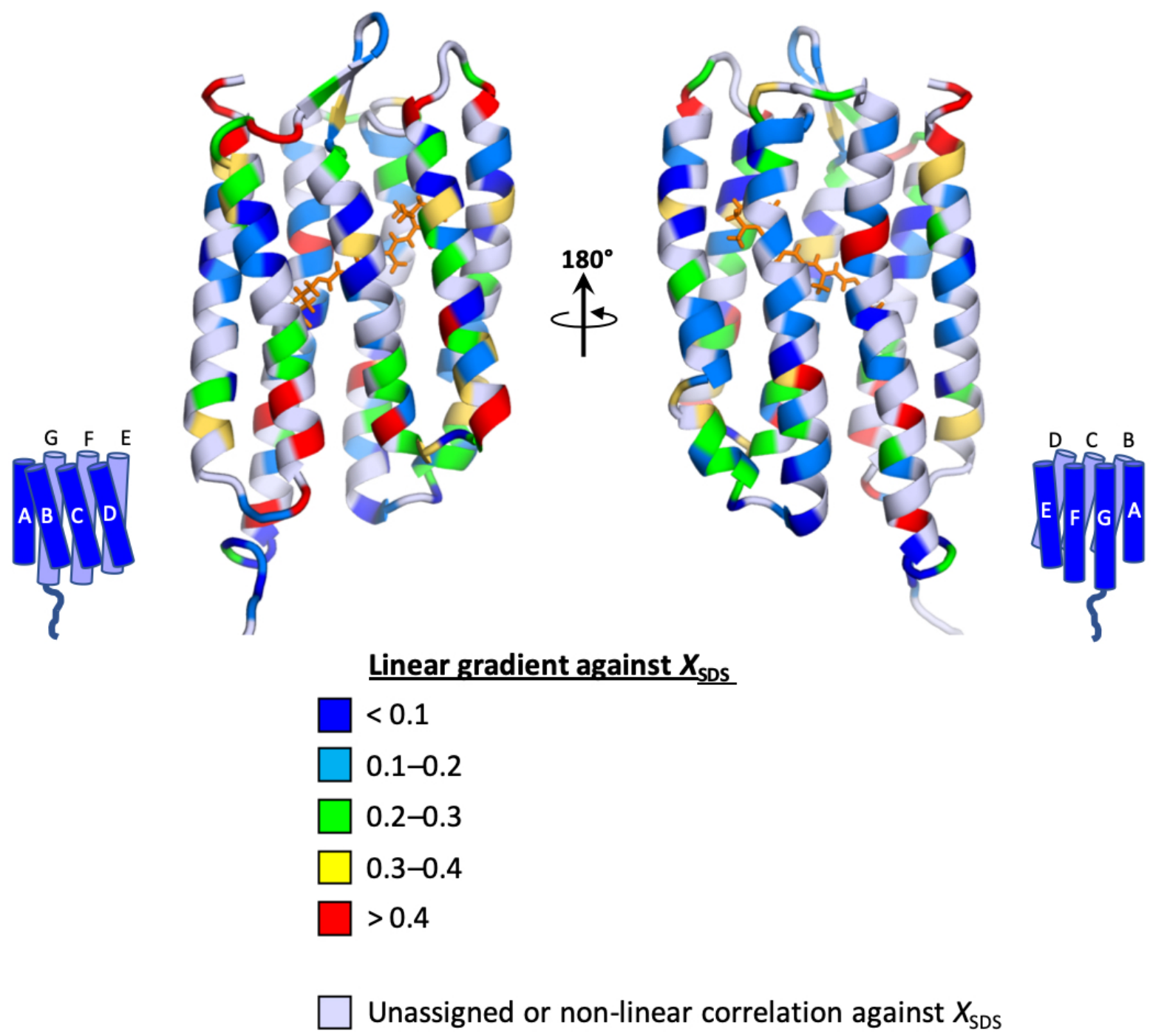

Figure S3: Residues with linear correlation between $\Delta \delta\left({ }^{1} \mathrm{H},{ }^{15} \mathrm{~N}\right)$ and $X_{\text {SDS }}$ Residues are coloured according to the slope of the linear correlation. 
(a)

(b)
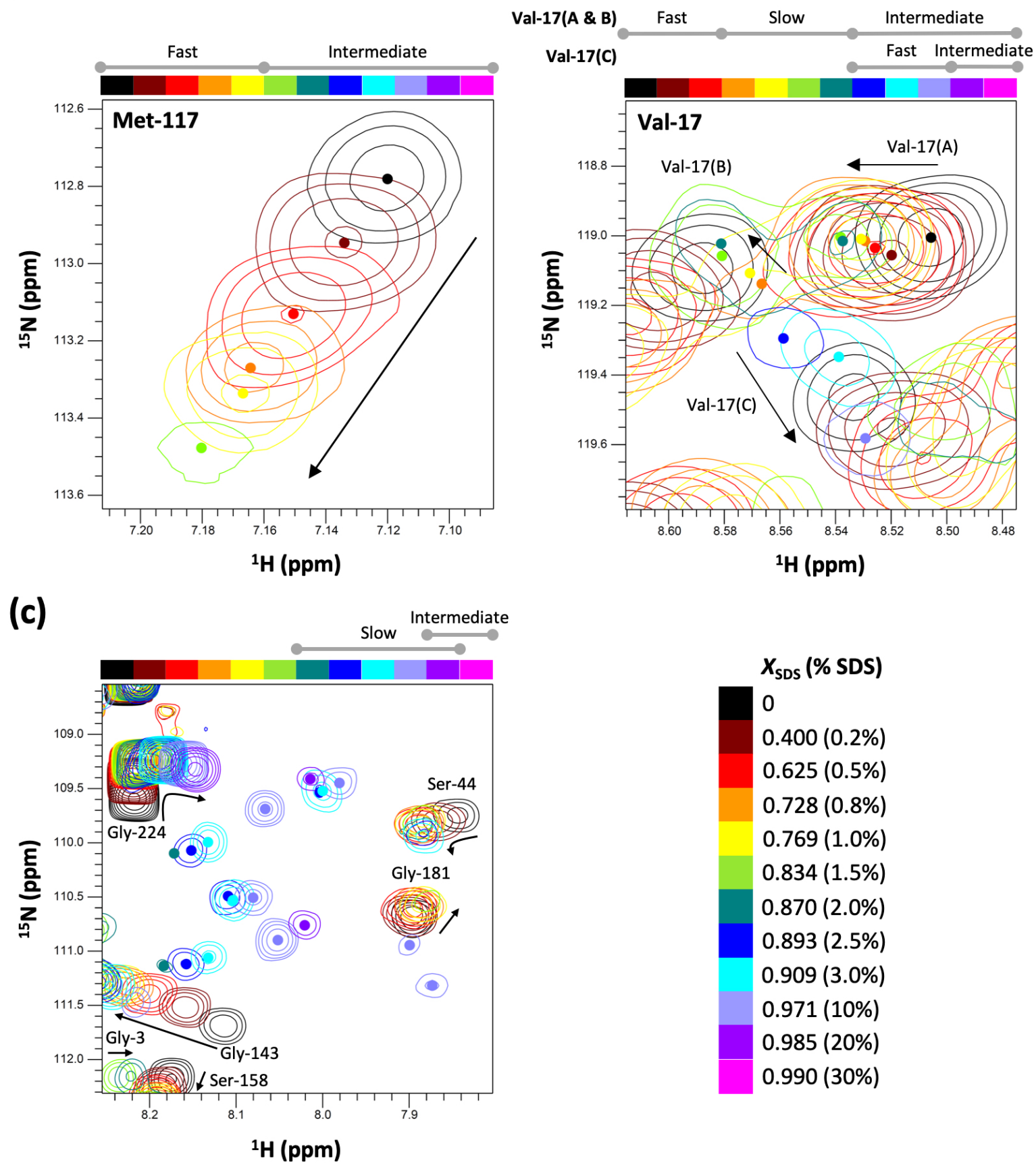

Figure S4: Examples of residues showing changes in conformational exchange regime with increasing $X_{\text {SDS }}$

Resonances of interest are marked with dots at the center of the peak. Chemical shift changes are represented using black arrows. (a) Met-117 shows intermediate timescale exchange in $>0.834 X_{\text {SDS }}$. (b) Conformers $\mathrm{A}$ and $\mathrm{B}$ of Val-17 backbone amide show transition from fast exchange in $0 X_{S D S}$ to slow exchange in $0.728-0.870 X_{\text {SDS }}$ to intermediate exchange in $>0.870 X_{S D S}$. Conformer $C$ dominates in $>0.893 X_{\text {SDS, }}$ and shows transition from fast exchange to intermediate exchange in $>0.985 X_{\text {SDS }}$. (c) Several backbone amides show slow timescale conformational exchange and large chemical shift changes in high $X_{\mathrm{SDS}}$, leading to difficulties with resonance assignment. 
(a)

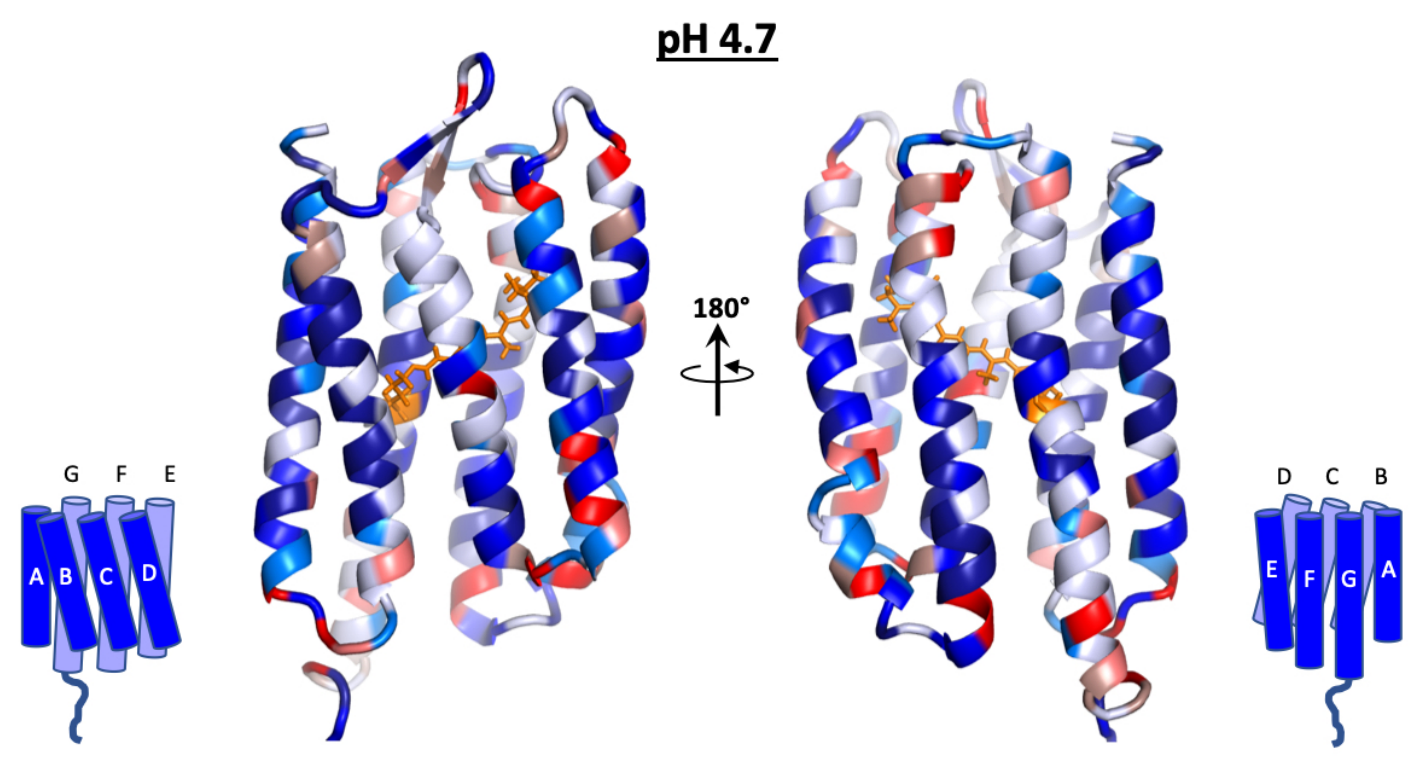

(b)

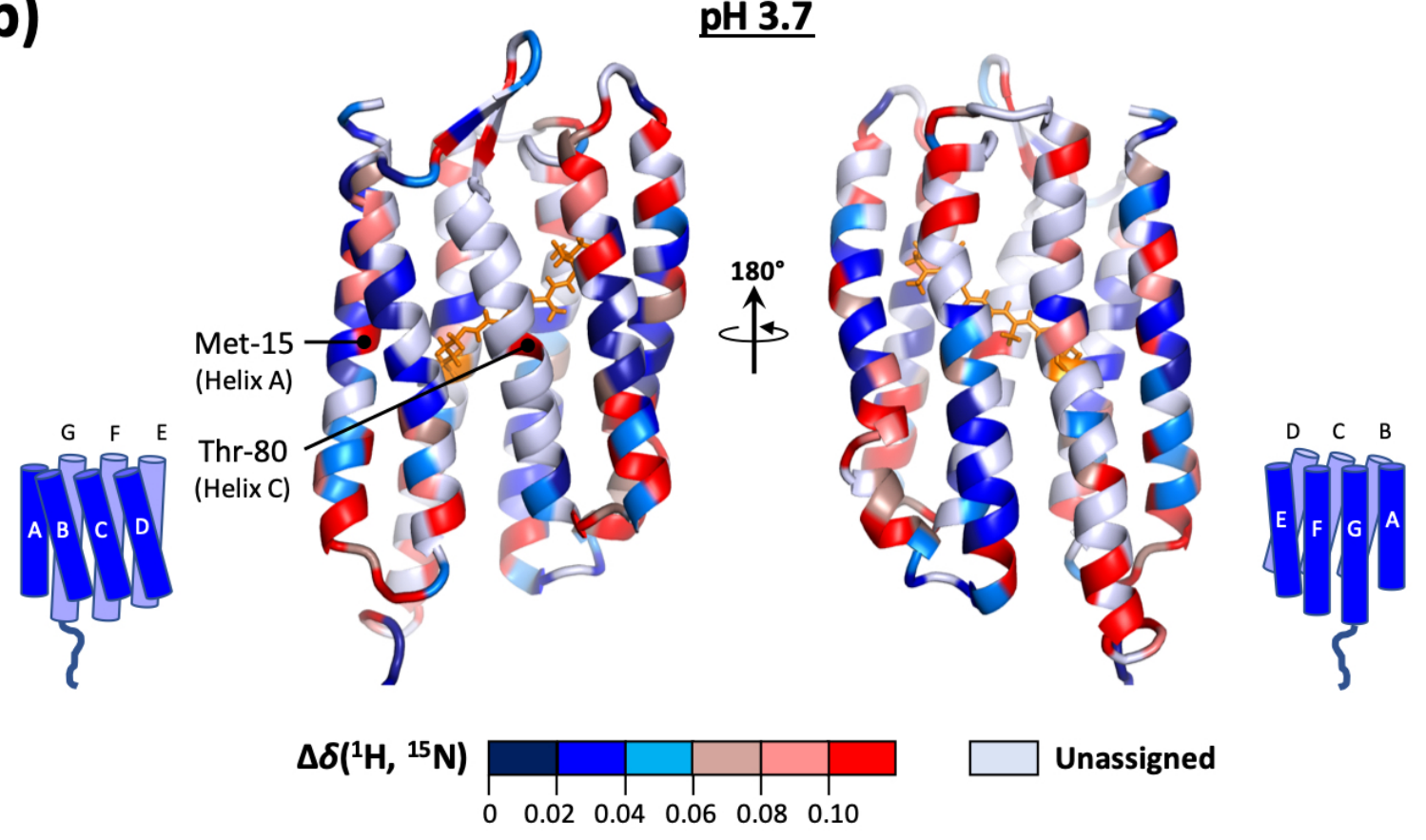

Figure S5: Chemical shift changes of pSRII at pH 4.7 and pH 3.7

$\Delta \delta\left({ }^{1} \mathrm{H},{ }^{15} \mathrm{~N}\right.$ ) (relative to chemical shifts at $\mathrm{pH} 6.0$ ) at (a) $\mathrm{pH} 4.7$ and (b) $\mathrm{pH} 3.7$ are mapped onto the pSRII structure. Residues are coloured from blue to red according to $\Delta \delta\left({ }^{1} \mathrm{H},{ }^{15} \mathrm{~N}\right)$ values, showing greater structural changes at the ends of helices than in the transmembrane region. Unassigned residues are coloured white. 
(a)

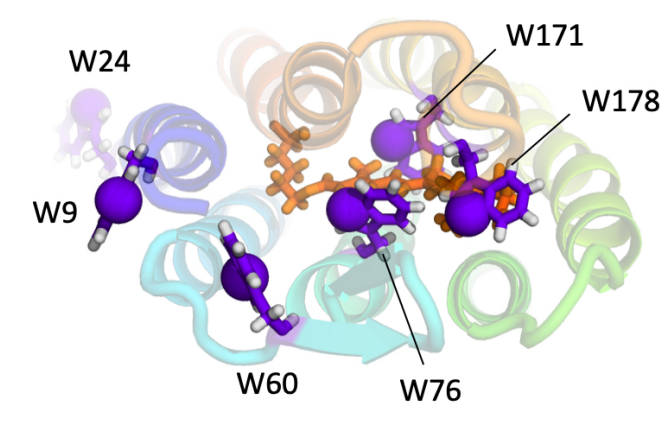

(c)

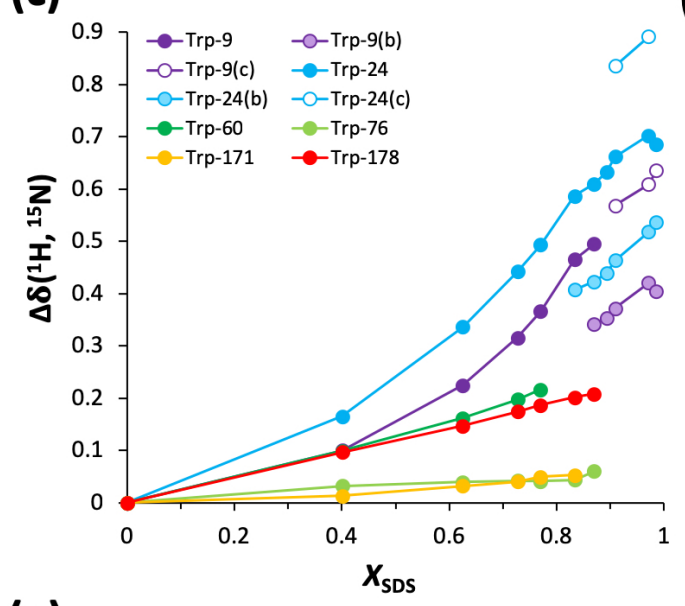

(e)

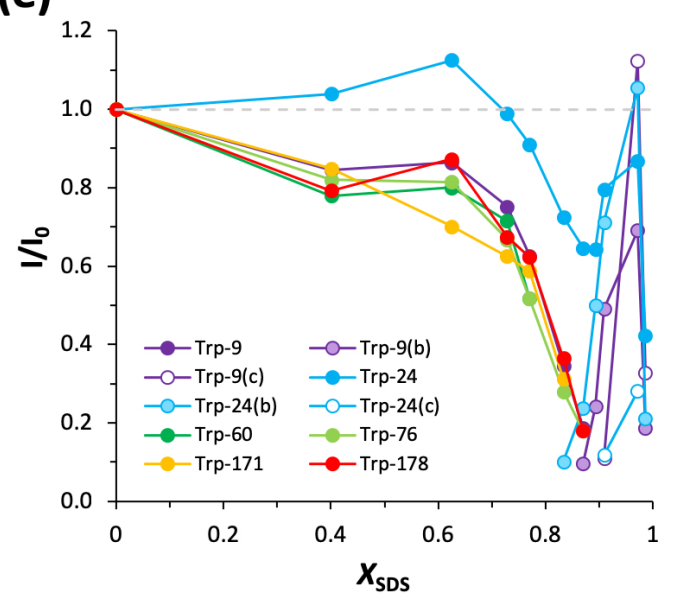

(b)

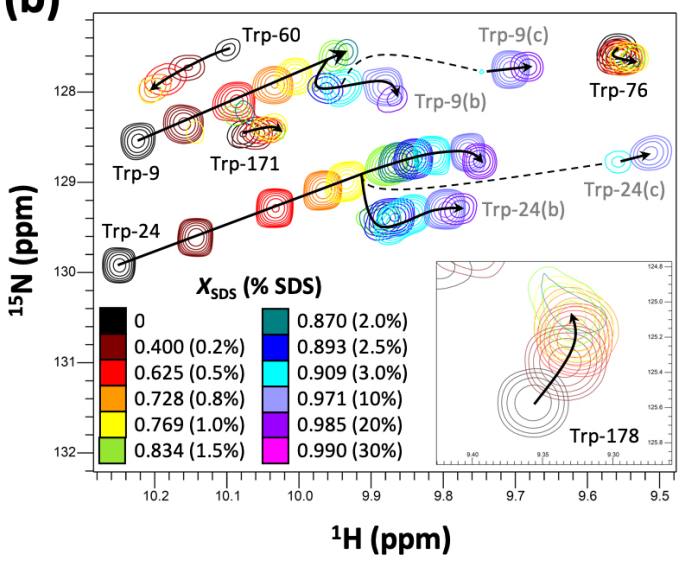

(d) (i) (ii)

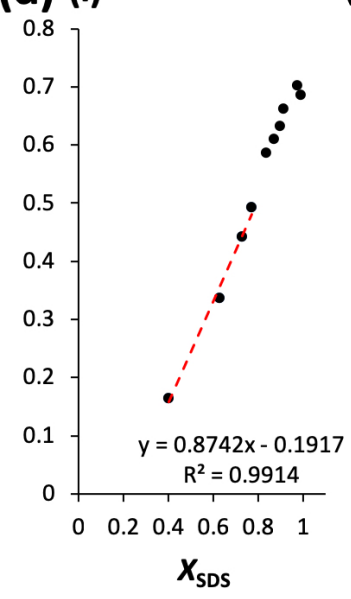

Figure S6: 2D $\left[{ }^{1} \mathrm{H},{ }^{15} \mathrm{~N}\right]$ BEST-TROSY of tryptophan side-chain indoles

(a) Tryptophan side-chains are shown as sticks, with the indole $\mathrm{N}$ atoms shown as spheres.

(b) Expanded view of the tryptophan side-chain indole region from an overlay of $2 \mathrm{D}\left[{ }^{1} \mathrm{H},{ }^{15} \mathrm{~N}\right]$ BEST-TROSY spectra recorded in different $X_{\text {SDS. }}$. Chemical shift changes are indicated by black arrows, with large shift changes represented using dashed lines. Alternative conformations (labelled as b and c) are observed for Trp-9 and Trp-24 side-chain indoles. Inset shows chemical shift changes for Trp-178 side-chain indole. (c \& e) $X_{\text {sDs }}$-dependence of $\Delta \delta\left({ }^{1} \mathrm{H},{ }^{15} \mathrm{~N}\right.$ ) (c) and $I / I_{0}$ (e) for tryptophan side-chain indoles. (d) Linear dependence of Trp-24 against (i) $X_{\text {SDS }}$ and (ii) absolute concentration of SDS. The fitted equations and goodness-of-fit $\left(R^{2}\right)$ of the linear regressions are shown in the bottom right corners of (i) and (ii). 